\title{
Does evolution lead to maximizing behavior?
}

\author{
Laurent Lehmann, ${ }^{1,2}$ Ingela Alger, ${ }^{3}$ and Jörgen Weibull ${ }^{4}$ \\ ${ }^{1}$ Department of Ecology and Evolution, University of Lausanne, Switzerland \\ ${ }^{2}$ E-mail: laurent.lehmann@unil.ch \\ ${ }^{3}$ Toulouse School of Economics and Institute for Advanced Study in Toulouse, Toulouse, France \\ ${ }^{4}$ Stockholm School of Economics, Stockholm, Sweden, and Institute for Advanced Study in Toulouse, Toulouse, France
}

Received January 15, 2015

Accepted June 1, 2015

\begin{abstract}
A long-standing question in biology and economics is whether individual organisms evolve to behave as if they were striving to maximize some goal function. We here formalize this "as if" question in a patch-structured population in which individuals obtain material payoffs from (perhaps very complex multimove) social interactions. These material payoffs determine personal fitness and, ultimately, invasion fitness. We ask whether individuals in uninvadable population states will appear to be maximizing conventional goal functions (with population-structure coefficients exogenous to the individual's behavior), when what is really being maximized is invasion fitness at the genetic level. We reach two broad conclusions. First, no simple and general individual-centered goal function emerges from the analysis. This stems from the fact that invasion fitness is a gene-centered multigenerational measure of evolutionary success. Second, when selection is weak, all multigenerational effects of selection can be summarized in a neutral type-distribution quantifying identity-by-descent between individuals within patches. Individuals then behave as if they were striving to maximize a weighted sum of material payoffs (own and others). At an uninvadable state it is as if individuals would freely choose their actions and play a Nash equilibrium of a game with a goal function that combines self-interest (own material payoff), group interest (group material payoff if everyone does the same), and local rivalry (material payoff differences).
\end{abstract}

KEY WORDS: inclusive fitness, uninvadable, game theory, maximizing behavior, Nash equilibrium.

\begin{abstract}
Individuals do not consciously strive to maximize anything; they behave as if maximizing something. It is exactly the same "as if" logic that we apply to "intelligent genes." Genes manipulate the world as if striving to maximize their own survival. They do not really "strive," but in this respect they do not differ from individuals. Dawkins (1982, p. 189)
\end{abstract}

The fundamental unit of behavior in the life and social sciences is the action. In decision theory (e.g., Kreps 1988; Binmore 2011), an individual's behavior is modeled as a choice of action or sequence of conditional actions from a set of feasible actions. This choice is guided by a striving to maximize some goal function, such as, for instance, one's own material well-being, or some altruistic or spiteful goal. The outcome of an individual's choice in general also depends on (perhaps random) events in the individual's environment, in which case the individual is assumed to strive to maximize the expected value of its goal function. In many, if not most cases, the environment partly consists of other decision makers, equipped with their feasible action sets and goal functions. Then, the expectation is also taken with respect to others' choice of action, which in turn may depend on those other individuals' expectations about "our" decision-maker's choice. Such interdependent decision problems are called games, and an individual's plan for what action to take under each and every circumstance that can arise in the interaction is then called a strategy for that player. A collection or profile of strategies, one for each individual, is a (Nash) equilibrium if no individual can increase its goal function value by a unilateral change of its strategy.

A long-standing question in evolutionary biology is whether natural selection leads individual organisms to behave as if they were maximizing some goal function. Because resources are 
limited, the material consequences for, and hence fitness of, one individual usually depends not only on the individual's own actions, but also on the actions of others. It is thus as if the organisms were caught in a game. If the resulting behaviors can be interpreted as if each individual was choosing a strategy to maximize a goal function, this will be of importance for the understanding and prediction of behavior. This is not only of interest to biology, but also to the social sciences, and in particular to economics, which is largely built on the supposition that individual behavior can be explained as the outcome from maximization of the expected value of some goal function.

In early evolutionary biology, the question of maximizing behavior was addressed by way of investigating optimality properties of mean fitness (defined as mean fertility or survival) under allele frequency change (Fisher 1930; Wright 1942; Kingman 1961). The underlying scheme was that natural selection invariably increases mean fitness and thus evolves individuals to express optimal actions given current environmental conditions. This has typically been investigated in settings with no social interactions (Wright 1942; Kingman 1961), that is, where the fitness of an individual does not depend on others' actions. Although in this case natural selection leads to an increase in mean fitness in the one locus case, it does often not do so in the multilocus case (Moran 1964; Ewens 2004, 2011). This suggests a priori that individuals are unlikely to behave as if they maximized their fitness.

For social interactions, Hamilton (1964) proved that mean inclusive fitness increases under additive gene action in a population under allele frequency change. Organisms should thus evolve to behave in such a way that their inclusive fitness is maximized. Hamilton's (1964) concept of inclusive fitness is based on a measure of fitness that is ascribed to a genotype or an allele (Hamilton 1964, p. 6). The inclusive fitness of an allele at a particular gene locus is the heritable part of the fitness of an average carrier of that allele, but where the source of variation of that fitness is decomposed into the effect of the allele in the carrier and that in other individuals from the population, hence the term “inclusive." Inclusive fitness is frequency independent under additive gene action and weak selection (although Hamilton's 1964 model allows to capture strategic interactions arising from interdependency in actions, see Rousset 2004). But this will generally be obtain as selection can be frequency-dependent at the genetic level at a given locus. Hence, even in the one-locus case it is not generally true that natural selection results in individuals behaving as if they strived to maximize their inclusive fitness (sensu Hamilton 1964).

One fundamental message of the population-genetic assessment of optimization under allele frequency change (Moran 1964; Ewens 2004, 2011) is that fitness maximization does not in general lead to individual-centered maximizing behavior under short-term evolution. However, concepts of fitness maximization can never- theless be illuminating under long-term evolution because they then allow characterization of evolutionarily stable states (Eshel 1991, 1996; Eshel et al. 1998). It is indeed well-established that the maximization of the growth rate of a nonrecombining heritable trait (here taken to be a gene) when rare-invasion fitnessprovides a condition of uninvadability of a mutant allele in a resident population, and this is a defining property of an evolutionarily stable state (Eshel 1983; Ferrière and Gatto 1995; Eshel et al. 1998; Rousset 2004; Metz 2011).

Because different alleles have different phenotypic effects (e.g., result in different streams of actions), the range of such effects can be conceived as the effective strategy space of the "strategic gene" (Haig 1997, 2012). From a gene's perspective (Dawkins 1978), invasion fitness can be regarded as the goal function a gene is striving to maximize. This is quite distinct from the goal function (if any such exists) that is to explain an individual's behavior, who can potentially interact with all others in the population. In order to answer the question as to whether at an evolutionary uninvadable state each individual appears to behave as if it were striving to maximize some individual-centered goal function, it is necessary to establish a link between invasion fitness (gene-centered perspective) and individual maximizing behavior (individual-centered perspective).

We are certainly not the first to explore these links. However, we feel that no previous study in this area has attempted to integrate evolutionary population dynamics, game theory, and behavioral ecology in such an exploration. In particular, dynamic conditions under which strategically interacting individual organisms behave as if they were maximizing some goal function in spatially structured populations appear to be lacking in three respects.

First, research in theoretical biology on the link between invasion fitness and maximizing behavior usually restricts attention to situations where the effect of an individual's action on the reproduction or survival of others does not depend on the actions of any other individual (Grafen 2006), or else it does not make this restriction, but assumes that an individual's goal is not affected by others' actions (Gardner and Welch 2011; Lehmann and Rousset 2014). Noninterdependence of effects of actions on survival and reproduction holds in interactions in which the effects of actions on the fitness of others are additively separable, or more generally, in what we here call strategically neutral games (Alger and Weibull 2012). This is, arguably, a rare case in practice. Although work on the relation between evolution and maximizing behavior in economics emphasizes the strategic interdependency of actions (e.g., Bergstrom 1995; Dekel et al. 2007; Heifetz et al. 2007; Alger and Weibull 2013), it is generally based on panmictic population assumptions, although natural populations tend to exhibit limited dispersal (Clobert et al. 2001), and this critically affects invasion fitness (e.g., Nagylaki 1992; Rousset 2004). 
Second, even though spatial population structure is usually taken into account in evolutionary biology, previous work on maximizing behavior often endorsed a concept of uninvadability derived from natural selection over only a single demographic (or reproductive) time period, the initial period in which the mutant arises (e.g., Grafen 2006, p. 553). By considering only one single demographic time period, in isolation from all others, however, one is likely to miss out fluctuations of mutant frequency whose average over multiple periods leads to the build-up of genetic associations that determine invasion fitness. For instance, genetic relatedness between interacting individuals is generally a multigenerational measure of statistical association, which captures the average effects of (random) genetic drift and natural selection on mutant frequency change (Rousset 2004; Roze and Rousset 2008).

Finally, if organisms behave as if they maximized some goal function, does their "free choice" occur at the level of individual actions, sequence of (conditional) actions, or at the level of whole decision systems for taking actions? Although behavioral ecology tends to emphasize that natural selection leads organisms to have phenotypes that maximize fitness (Alcock 2005), it usually also emphasizes the constraints that endogenously link actions from one moment to the next (McNamara and Houston 1999; Fawcett et al. 2012), which precludes (the appearance of) free choice. It thus remains unclear in evolutionary biology at which phenotypic level maximizing behavior is generally conceived (if at all).

The aim of this article is to fill these gaps and to provide connections between (1) explicit population-dynamic evolutionary uninvadability, (2) game-theoretic equilibrium in strategic interactions between individuals, and (3) behavioral ecology formulations of behavior under different constraints. To that aim, we develop a mathematical model of multimove strategic interactions and evolution in a spatially structured population, within which we formalize notions of personal fitness and invasion fitness, and derive from them goal functions that individual organisms will, through their behavior, appear to be maximizing, while what is in fact being maximized is invasion fitness.

The rest of the article is organized as follows. First, we present a multimove model of behavior under social interactions based on the state-space approach of behavioral ecology (McFarland and Houston 1981; Enquist and Ghirlanda 2005), and define uninvadability of mutant behavior by building on established results for the invasion of single mutant types in spatially structured population (in particular the branching-process approach of Wild 2011, which subsumes invasion fitness as given by the expected number of successful emigrants produced by a single immigrant; Metz and Gyllenberg 2001; Ajar 2003). Second, we connect behavioral ecology formulations of behavior to the standard game-theoretic concept of behavior (Fudenberg and Tirole 1991; Osborne and Rubinstein 1994), and postulate two goal functions for social interactions that are well anchored in the social evolution and inclusive fitness literature. This allows us to state the "as if" question in terms of well-established game theoretic concepts. We show that neither of the two goal functions leads to general equivalence between uninvadability and maximizing behavior.

Then, we turn to the analytically less forbidding case of weak selection and suggest a third goal function, which has not been studied before. Here, we establish a positive result and show that maximizing behavior under this goal function is indeed equivalent with uninvadability under essentially all conditions on behavior in social interactions. Finally, we discuss our results and interpretation in terms of maximizing behavior under behavioral constraints.

\section{Model \\ BIOLOGICAL ASSUMPTIONS}

\section{Life cycle}

We consider a population of haploid individuals structured into an infinite number of patches (or islands), each subject to exactly the same environmental conditions and consisting of exactly $N$ adult individuals (i.e., Wright's 1931, infinite island model of dispersal). The life cycle of individuals in this population consists of the following events that occur over one demographic time period. (1) As an outcome of social interactions with others, each adult individual produces asexually a large number of offspring and then either survives into the next demographic time period or dies with a probability independent of age. (2) Each offspring either disperses or remains in its natal patch, and each migrant disperses to a uniformly randomly chosen non-natal patch. (3) In each patch the random number of aspiring offspring, some native and some immigrant from other patches, compete for the breeding spots vacated by the death of adults. In each patch exactly $N$ individuals survive this density-dependent competition.

We assume that the probability for an offspring to migrate is always positive. No other assumption about fecundity, survival, migration, or competition is made at this stage of the analysis. In particular, the demography allows for exactly one, several, or all adults to die per demographic time unit (overlapping and nonoverlapping generation models).

\section{Behavior in social interactions}

We envision social interactions as being extended over social time periods although they take place within one single demographic time period (stage [1] in the life cycle until [2] starts). We thus deal with two time scales, one (slow) for the demographic events, and one fast for social interactions taking place over possibly several social time periods. The model thus covers both one-shot and repeated interactions. 
Table 1. Formal definition of the main functions and notation for vectors.

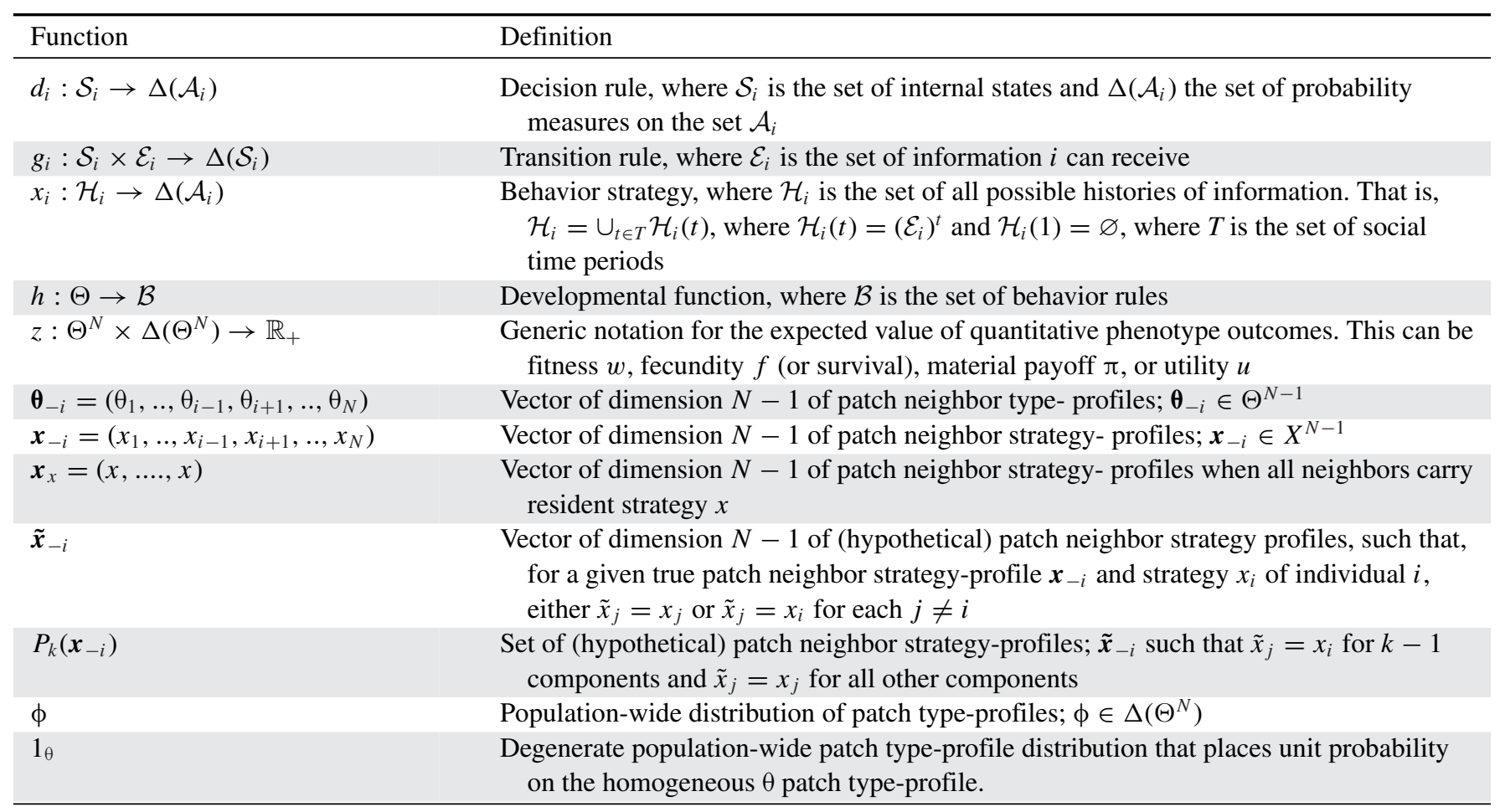

Following established lines in behavioral ecology (e.g., McFarland and Houston 1981; Leimar 1997; Enquist and Ghirlanda 2005), we take the action (e.g., a motor pattern, a signal, or a transfer of resources to a neighbor) as the fundamental behavioral unit by which an individual interacts with others in each social period. The set of feasible actions available to individual $i$ (where $i \in\{1,2, \ldots, N\}$ ) in a given patch is denoted $\mathcal{A}_{i}$, and the action taken by that individual at social time $t$,

$$
a_{i}(t)=d_{i}\left(s_{i}(t)\right),
$$

is assumed to be determined by the individual's internal state $s_{i}(t)$, which belongs to the set $\mathcal{S}_{i}$ of internal states that an individual can be in, and $d_{i}$ is its decision rule (see Table 1 for a formal definition of functions and notations).

An individual's (internal) state changes (possibly stochastically) over time, and the state of individual $i$ at any time $t>1$,

$$
s_{i}(t)=g_{i}\left(s_{i}(t-1), e_{i}(t-1)\right),
$$

is assumed to be determined by the individual's state $s_{i}(t-1)$ in the previous social time period and the information $e_{i}(t-1)$ obtained during that time period, where $\mathcal{E}_{i}$ is the set of information it can receive. This information could consist of any more or less noisy private or public signals about the individual's own action and/or that of others. A simple example is the (public) profile of actions $e_{i}(t)=\left(a_{1}(t-1), . ., a_{N}(t-1)\right)$ taken by all individuals in individual $i$ 's patch, but the information could also consist of the actions taken by individuals on other patches.
We call the tuple $b_{i}=\left(d_{i}, g_{i}, s_{i}(1), \mathcal{A}_{i}, \mathcal{S}_{i}, \mathcal{E}_{i}\right)$ the behavior rule of individual $i$. This rule defines how the individual acts and reacts to others in the sequence of social interactions within one demographic time period. When the set of internal states is finite (infinite), a behavior rule is a finite (infinite) state machine (Minsky 1967), and as such neural networks or universal Turing machines can be implemented by a behavior rule (Haykin 1999).

\section{Individual types and personal fitness}

We assume that the behavior rule of an individual is fixed at birth and determined by its type, which is inherited faithfully from its parent. The set of admissible types is denoted $\Theta$ and $\theta_{i}$ denotes the type of individual $i$, which determines its behavior rule by way of the developmental function $h: b_{i}=h\left(\theta_{i}\right)$ (Table 1). Because interactions, in general, may occur between individuals from the same or different patches, any phenotype of an individual may depend not only on its own type, but also on the types of its patch neighbors and the types of individuals taken at large from the population. For instance, the survival or the actions expressed by individual $i$ in the social interactions may depend on the types of others because these actions depend on the state the individual is in, which itself is a function of environmental information (eqs. 1 and 2).

A fundamental feature of the infinite island model is that the phenotypic effects of individuals in other patches on a given individual is, by the law of large numbers, nonstochastic, however, and depends only on averages (Chesson 1981). This implies that a given phenotype of an individual can be expressed as a function 
of the individual's own type, the type profile of its patch neighbors, and the distribution of type profiles across all patches in the population at large. One such phenotype that will play a fundamental role in our analysis is the personal fitness of an individual, which we define as an individual's expected number of surviving descendants (possibly including the individual itself, for demographics where adults may survive) after one demographic time period. In the island model, this can then be written for individual $i$ as $w\left(\theta_{i}, \boldsymbol{\theta}_{-i}, \phi\right)$, where $\theta_{i}$ is the individual's type, $\boldsymbol{\theta}_{-i}$ is the patch neighbor type profile (thus excluding $i$ 's type, see Table 1), and $\phi$ is the population-wide distribution of patch type-profiles.

Due to migration and competition for breeding spots, an individual's personal fitness will in general depend on the vital rates (fecundity and survival) and migration rates among its patch neighbors and in the population at large (e.g., Frank 1998; Rousset 2004). Fitness then depends on vital rates (see Box 1 for an example), and these in turn depend on the behaviors during the social interactions, which in turn depend on the behavior rules of population members, so that, ultimately, fitness depends on the distribution of (mutable) types in the population (see Fig. 1).

Box 1. Individual fitness under a Moran process. An example of a fitness function for the island model can be obtained by assuming a Moran process (Ewens 2004), where exactly one randomly sampled adult on each patch dies per demographic time period (Mullon and Lehmann 2014). Assuming that all offspring have the same migration probability $m$, the fitness of a focal individual $i$ is given by:

$$
\begin{aligned}
w\left(\theta_{i}, \boldsymbol{\theta}_{-i}, \phi\right)= & 1-\frac{\mu\left(\theta_{i}, \boldsymbol{\theta}_{-i}, \phi\right)}{\sum_{j=1}^{N} \mu\left(\theta_{j}, \boldsymbol{\theta}_{-j}, \phi\right)} \\
& +\frac{1}{N}\left[\frac{(1-m) f\left(\theta_{i}, \boldsymbol{\theta}_{-i}, \phi\right)}{(1-m) \frac{1}{N} \sum_{j=1}^{N} f\left(\theta_{j}, \boldsymbol{\theta}_{-j}, \phi\right)+m \bar{f}(\phi)}\right. \\
& +m f\left(\theta_{i}, \boldsymbol{\theta}_{-i}, \phi\right) \\
& \left.\times \int_{\Theta^{N}} \frac{1}{(1-m) \frac{1}{N} \sum_{h=1}^{N} f\left(\theta_{h}, \boldsymbol{\theta}_{-h}, \phi\right)+m \bar{f}(\phi)} \phi(\mathrm{d} \boldsymbol{\theta})\right] .
\end{aligned}
$$

The first two terms constitute the part of fitness stemming from own survival; that is, it represents the probability of survival, where the death probability is of the form $\mu\left(\theta_{i}, \boldsymbol{\theta}_{-i}, \phi\right) /\left[\sum_{j=1}^{N} \mu\left(\theta_{j}, \boldsymbol{\theta}_{-j}, \phi\right)\right]$, where $\mu\left(\theta_{i}, \boldsymbol{\theta}_{-i}, \phi\right)$ is the death-factor for individual $i$. The third term, with square brackets, is the part of fitness stemming from settlement of offspring in vacated breeding spots. This depends on the fecundity $f\left(\theta_{i}, \boldsymbol{\theta}_{-i}, \phi\right)$ of individual $i$ (defined as its expected number of offspring produced in a demographic time unit) and also on the average fecundity in the population as a whole, $\bar{f}(\phi)=\int_{\Theta^{N}}\left[\frac{1}{N} \sum_{h=1}^{N} f\left(\theta_{h}, \boldsymbol{\theta}_{-h}, \phi\right)\right] \phi(\mathrm{d} \boldsymbol{\theta})$, where, for any patch-profile $\boldsymbol{\theta}=\left(\theta_{1}, . ., \theta_{N}\right) \in \Theta^{N}$, the integrand is the av- erage fecundity of the $N$ individuals in the patch. The first term inside the square brackets in equation (B-a) is the individual's fitness through its philopatric offspring, each of whom competes for the local vacated breeding spot with all philopatric offspring from the same patch and with all migrating offspring from other patches who also aspire for this breeding spot. The second term inside the square brackets is the focal individual's fitness stemming from its dispersing progeny to other patches.

The fecundity $f\left(\theta_{i}, \boldsymbol{\theta}_{-i}, \phi\right)$ and death factors $\mu\left(\theta_{i}, \boldsymbol{\theta}_{-i}, \phi\right)$ are vital rates that depend on the material payoff obtained in social interactions, where the material payoff depends on the stream of actions taken over all social time periods. These actions in turn depend on the behavior rules of interacting individuals and ultimately on their types. This hierarchical dependence is illustrated in Figure 1.

We will assume, for analytical tractability, that any phenotype of an individual is expressed unconditionally on age (neither their own nor others') and does thus not vary with demographic time (above and beyond variations due to changes in the type distribution). Recalling that all individuals are subject to the same environmental conditions (i.e., there is no class structure, Taylor 1990), any nonmutable phenotype of a given individual $i$, such as for instance fitness $w\left(\theta_{i}, \boldsymbol{\theta}_{-i}, \phi\right)$, can then be considered to be invariant under permutation of the elements of the type profile $\boldsymbol{\theta}_{-i}$ of its patch neighbors, and this symmetry across neighbors will be assumed throughout.

\section{UNINVADABILITY}

Suppose that initially the population is monomorphic for some resident type $\theta$ and that a single individual mutates to some new type $\tau$. Will this mutant type "invade" the population? If the resident type $\theta$ is such that any mutant type $\tau \in \Theta$ goes extinct with probability one, we will say that $\theta$ is uninvadable. Uninvadability could also informally be thought of as evolutionary stability as it is similar to the verbal definition of the latter (Maynard Smith and Price 1973). But this terminology will not be used here, because it differs from the formal definition of evolutionary stability (Maynard Smith and Price 1973), which subsumes that an evolutionarily stable state should be an attractor of the evolutionary dynamics.

In order to get a grip on uninvadability, consider a single individual of type $\theta^{\prime} \in\{\tau, \theta\}$ in one patch, to be called the focal patch, in a population that is otherwise monomorphic for type $\theta$. Through reproduction, this individual may found a lineage with local descendants and, through migration, descendants reaching adulthood in other patches. Owing to our life cycle assumptions, the probability that the offspring of a migrant descendant of the lineage-founding individual will migrate back to the focal patch is zero. As a consequence, the lineage descending from the initial 


$\begin{array}{llcl}\text { Types } & \text { Action streams } & \text { Vhavior rules } & \text { Vital rates }\end{array}$

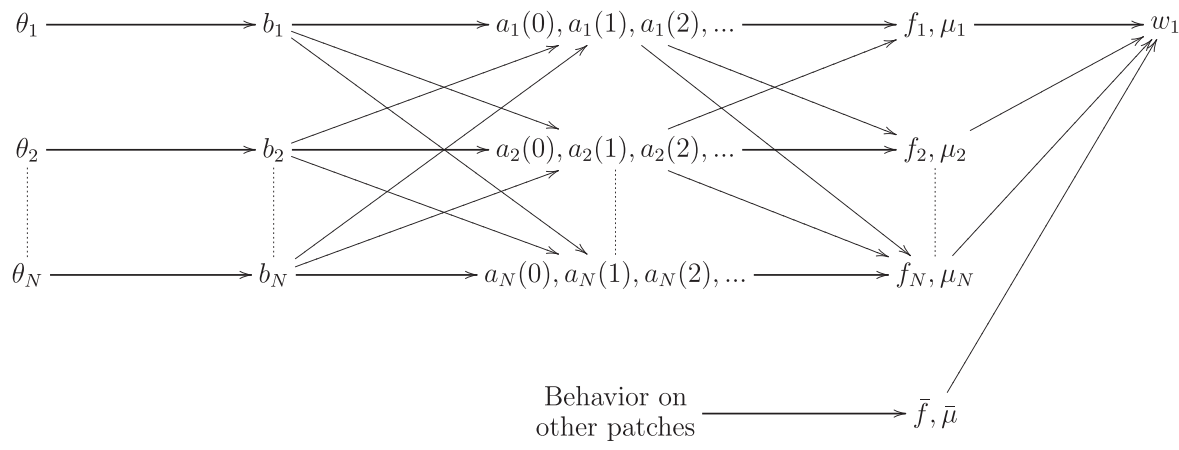

Figure 1. Illustration of the dependence of the fitness of a focal individual (here individual 1) on a focal patch on the various components of the model in the island model of dispersal. Fitness, $w$, is a function of the vital rates of patch members such as fecundity, $f$, or death factor $\mu$, and on the vital rates of individuals from other patches. Vital rates in turn depend on the behaviors of all individuals, more exactly on the action streams, $a(1), a(2), a(3) \ldots$, during the social time period. The action streams are determined by the behavior rules, $b$, of individuals, and the behavior rules themselves depend on evolvable (potentially multidimensional) traits, the individual's type, $\theta$.

founder will eventually go extinct locally and the population can be taken to be virtually monomorphic for $\theta$.

With this assumption, we can define the lineage fitness of a type $\theta^{\prime} \in\{\tau, \theta\}$ as

$$
W\left(\theta^{\prime}, \theta\right)=\sum_{k=1}^{N} \sum_{\boldsymbol{\theta}_{-i} \in S_{k}\left(\theta^{\prime}, \theta\right)} w\left(\theta^{\prime}, \boldsymbol{\theta}_{-i}, 1_{\theta}\right) q_{k}\left(\theta^{\prime}, \theta\right) .
$$

This is the average personal fitness of a randomly drawn member of the local lineage, where the average is taken over the (finite) lifespan of this local lineage when the population is otherwise monomorphic for type $\theta$. Lineage fitness depends on three quantities. First, $w\left(\theta^{\prime}, \boldsymbol{\theta}_{-i}, 1_{\theta}\right)$ is the personal fitness of a member $i$ of the local lineage, whose type is $\theta^{\prime}$, on a patch with neighbor typeprofile $\boldsymbol{\theta}_{-i}$, when the population at large is monomorphic in type $\theta$, for which case the patch type-profile distribution is denoted $1_{\theta}$. Second, $q_{k}\left(\theta^{\prime}, \theta\right)$ is the probability that the neighbor type-profile $\boldsymbol{\theta}_{-i}$ of a given focal individual of type $\theta^{\prime}$ will consist of exactly $k-1$ other local lineage members (see Box 2 for details and on how the associated probability distribution allows us to define relatedness between patch members). Finally, $S_{k}\left(\theta^{\prime}, \theta\right)$ is the set of neighbor type-profiles $\boldsymbol{\theta}_{-i}$ such that exactly $k-1$ neighbors are also members of the local lineage.

A necessary and sufficient condition for a type $\theta$ to be uninvadable is that no mutant type $\tau$ has a lineage fitness above that of the resident, in other words it should solve the maximization problem

$$
\max _{\tau \in \Theta} W(\tau, \theta) .
$$

This shows that lineage fitness is a measure of invasion fitness, which takes into account all consequences of the discrete and finite nature of patches on selection (see proof in Appendix SA). In particular, $W(\tau, \theta)-W(\theta, \theta)$ has the same sign as the growth rate of a mutant when rare in the population, which is the usual and general measure of invasion fitness (Ferrière and Gatto 1995; Caswell 2000; Metz 2011).

Now that we have a grip on how to evaluate the uninvadability of types, and therefore also of behavior rules, we proceed to define maximizing behavior.

Box 2. Local type-profile distribution and relatedness. The type-profile distribution in equation (3) is given by:

$$
q_{k}\left(\theta^{\prime}, \theta\right)=\left(\begin{array}{c}
N-1 \\
k-1
\end{array}\right)^{-1} p_{k}\left(\theta^{\prime}, \theta\right)
$$

where the factorial accounts for all the ways that a profile of length $N-1$ can contain $k-1$ lineage members, and

$$
p_{k}\left(\theta^{\prime}, \theta\right)=\frac{k t_{k}\left(\theta^{\prime}, \theta\right)}{\sum_{h=1}^{N} h t_{h}\left(\theta^{\prime}, \theta\right)}
$$

is the probability, for a randomly drawn local lineage member, to have $k-1$ other local lineage members. This depends on the expected sojourn time $t_{k}\left(\theta^{\prime}, \theta\right)$ (number of demographic time periods) the lineage consists of $k \in\{1, \ldots, N\}$ members. This sojourn time is finite because the size of the local lineage founded by a single founder has exactly one absorbing state, namely, the extinction of the local lineage $(k=0)$ when migration is positive (see Appendix SD for details). Equation (B-c) thus gives the experienced lineage-size distribution; the probability distribution of 
lineage size - the number of individuals who are identical by descent-as experienced by a randomly drawn lineage member.

A standard statistic representing the magnitude of identity-by-descent is the pairwise relatedness between patch members (Wright 1931; Frank 1998; Rousset 2004). We here use the relatedness measure defined as:

$$
r\left(\theta^{\prime}, \theta\right)=\sum_{k=1}^{N} \frac{k-1}{N-1} p_{k}\left(\theta^{\prime}, \theta\right),
$$

which is the probability that a local lineage member's randomly drawn neighbor will also be a lineage member, that is, that they both descend from the founder of the local lineage. For $\theta^{\prime}=\theta$, the expression on the right-hand side of (B-d) boils down to the standard coefficient of relatedness evaluated in the neutral process - that is, when every individual has exactly the same fitness (e.g., Crow and Kimura 1970; Rousset 2004). For instance, for the Moran island model one obtains

$$
r(\theta, \theta)=\frac{1-m}{1-m+N m},
$$

which displays the canonical feature that relatedness is decreasing in the migration probability and in patch size (calculations for the Moran process are given in Appendix SD).

\section{MAXIMIZING BEHAVIOR}

A key step in our approach consists in noting that our definition of a behavior rule can implement that of a behavior strategy as defined in noncooperative game theory (see Fudenberg and Tirole 1991 or Osborne and Rubinstein 1994). To see the connection between behavior rules and behavior strategies, suppose that $g_{i}$ concatenates the most recent information to all previous information (by setting $s_{i}(1)=\varnothing$ and $s_{i}(t)=\left(s_{i}(t-1), e_{i}(t-1)\right)$ for all $t>1)$. Then, an individual's internal state $s_{i}(t)$ in social time period $t>1$ depends on the whole history of information up to time $t-1$, that is, $s_{i}(t)=\left(e_{i}(1), \ldots, e_{i}(t-1)\right)$ for $t>1$ [the whole history of actions if $\left.e_{i}(t)=\left(a_{1}(t-1), \ldots, a_{N}(t-1)\right)\right]$. In this case, the set of internal states is given by the set $\mathcal{H}_{i}$ of all possible histories of information available to individual $i$ [all possible histories of actions if $e_{i}(t)=\left(a_{1}(t-1), . ., a_{N}(t-1)\right)$, see Box 2 for details on $\mathcal{H}_{i}$ ]. We denote by $x_{i}$ the decision rule of individual $i$ in this specific case where the internal state records the whole history of information until the time point where a decision has to be taken (see Table 1). In game-theoretic terminology $x_{i}$ is a behavior strategy.

With this precise concept of a strategy $\left(x_{i}\right)$ in hand, we can formally represent interactions in the population at large as a game with infinitely many players, where all players use strategies from the same strategy set, denoted $X$. To this end, it is necessary to define each player's utility or goal function, that is, the function that describes the value that the player attaches to every possible strategy profile. Let $u\left(x_{i}, \boldsymbol{x}_{-i}, \phi\right)$ represent how individual $i$ values that particular strategy constellation $\left(x_{i}, \boldsymbol{x}_{-i}, \phi\right)$, where $x_{i}$ is its own strategy, $\boldsymbol{x}_{-i}$ is the strategy profile for its patch neighbors, and $\phi$ here stands (by slight abuse of notations) for the patchprofile distribution in the population at large. Thus, $u$ is a goal function for individual $i$ and we assume it to be symmetric in the same way as the fitness and fecundity functions are.

This setup then defines a symmetric normal-form game that we denote by $G=(\mathbb{N}, X, u)$, where the first item is the (infinite) set of players, the second item is the strategy set for each player, and the third item is each player's goal function $u$. A canonical concept for prediction of behavior in such a game is Nash equilibrium, strategy profiles in which no individual can get a higher utility by a unilateral deviation. We denote by $X_{\mathrm{E}}(u) \subseteq X$ the set of symmetric Nash equilibrium strategies (equilibria in which all players use the same strategy), and we will only consider such equilibria throughout. We then have that $x \in X_{\mathrm{E}}(u)$ if and only if $x_{i}=x$ solves the maximization problem

$$
\max _{x_{i} \in X} u\left(x_{i}, \boldsymbol{x}_{x}, 1_{x}\right),
$$

where $\boldsymbol{x}_{x}$ denotes the $(N-1)$ - dimensional vector whose components all equal $x$. In other words: if all other individuals in the population use strategy $x$, and individual $i$ was free to choose its strategy $x_{i}$ and its goal was to maximize the function $u$, then it would do the same as the others, that is, choose $x_{i}=x$. Thus, the strategies $x$ in the set $X_{\mathrm{E}}(u)$ are precisely those that are compatible with each individual maximizing its goal function when all the others use strategy $x$.

\section{THE "AS IF" QUESTION}

We are now ready to make a link with the evolutionary model presented above. To that end, suppose that there is a one-to-one correspondence between an individual's type and its strategy, that is, an individual's type directly determines its behavior strategy. Formally, let the set of types $\Theta$, on which natural selection operates, be the same as the set $X$ of behavior strategies, from which individuals make their choices.

Then, the personal fitness of $i$ writes $w\left(x_{i}, \boldsymbol{x}_{-i}, \phi\right)$. A strategy $x$ is then uninvadable if and only if satisfies the uninvadability maximization problem (eq. 4 with $\Theta=X$ ). Let $X_{\mathrm{U}}$ denote the set of uninvadable strategies. The "as if" question can then be stated as follows. Does there exist a goal function $u$ for which the set $X_{\mathrm{E}}(u)$ of (symmetric) Nash equilibrium strategies is the same as the set $X_{\mathrm{U}}$ of uninvadable strategies?

At first sight, it may seem that the lineage fitness function, $W(y, x)$ (see eq. 3), would fit the bill. However, this is not true because lineage fitness is a multigenerational measure of fitness and is a function of only two strategies, $y$ and $x$, whereas the "as 
if" question requires a goal function that depends on the whole population strategy-profile in a given generation. Nevertheless, lineage fitness $W$ can be thought of as the goal function of a focal "strategic gene" (Dawkins 1978; Haig 1997, 2012); that is, a gene attempting to maximize its own transmission across generations in a population where individuals behave according to the strategy of another gene, the focal's gene coplayer. According to this interpretation, strategy $x$ is uninvadable if and only if the type pair $(x, x)$ constitutes a Nash equilibrium in the symmetric two-player game in which strategies are elements of $X$ and the game payoff to a strategy $y$, when played against a strategy $x$, is lineage fitness $W(y, x)$.

Returning to the "as if" question, we consider two individualcentered goal functions that allow individuals to rank strategy constellations. First, we consider the goal function defined by

$u_{\mathrm{A}}\left(x_{i}, \boldsymbol{x}_{-i}, \phi\right)=w\left(x_{i}, \boldsymbol{x}_{-i}, \phi\right)+r(\bar{x}, \bar{x}) \sum_{j \neq i} w\left(x_{j}, \boldsymbol{x}_{-j}, \phi\right)$,

where $r(\bar{x}, \bar{x})$ is the pairwise relatedness between patch members (see Box 2) and $\bar{x}$ is the average strategy used in the population at large. This goal function is the individual's own personal fitness plus the personal fitness of all other individuals in the population weighted by their relatedness to the individual in question, and is in line with textbook representations of inclusive fitness (e.g., Alcock 2005) but is at variance with the concept of inclusive fitness described in Hamilton (1964).

The second individual-centered goal function that we consider is closer to the lineage-fitness function and defined by

$$
u_{\mathrm{B}}\left(x_{i}, \boldsymbol{x}_{-i}, \phi\right)=\sum_{k=1}^{N} \sum_{\tilde{\boldsymbol{x}}_{-i} \in P_{k}\left(\boldsymbol{x}_{-i}\right)} w\left(x_{i}, \tilde{\boldsymbol{x}}_{-i}, \phi\right) q_{k}(\bar{x}, \bar{x}),
$$

where the $q_{k}$ s are as defined in the lineage fitness function (eq. B-b in Box 2) and $P_{k}\left(\boldsymbol{x}_{-i}\right)$ is the subset of (hypothetical) patch neighbor strategy profiles $\tilde{\boldsymbol{x}}_{-i}$ such that exactly $k-1$ components of the (true) neighbor patch strategy profile $\boldsymbol{x}_{-i}$ have been replaced by $i$ 's strategy $x_{i}$, whereas the remaining $N-k$ components of $\tilde{\boldsymbol{x}}_{-i}$ are identical to those in $\boldsymbol{x}_{-i}$ (see Table 1). This goal function is thus the average personal fitness of individual $i$, where the weight attached to the event that $k-1$ neighbors use the same strategy as the individual itself is the probability that $k-1$ of a focal individual's neighbors belong to $i$ 's lineage, according to the experienced type-profile distribution in the evolutionary model.

\section{Uninvadability and Maximizing Behavior FIRST-ORDER CONDITIONS}

We are now in a position to relate the set of Nash equilibrium strategies, under each of the two goal functions, to the set of strategies that are uninvadable. To that end, we will start by considering the simple case where strategies can be represented as real numbers in some open set $X$. Owing to equation (4), any uninvadable such strategy $x \in X$ must then satisfy the first-order condition

$$
\begin{aligned}
\left.\frac{\partial W(y, x)}{\partial y}\right|_{y=x} & =w_{1}\left(x, \boldsymbol{x}_{x}, 1_{x}\right)+r(x, x)(N-1) w_{N}\left(x, \boldsymbol{x}_{x}, 1_{x}\right) \\
& =0
\end{aligned}
$$

where $w_{j}\left(x, \boldsymbol{x}_{x}, 1_{x}\right)$ is the partial derivative of $w\left(x_{i}, \boldsymbol{x}_{-i}, \phi\right)$ with respect to its $j$ th argument (for $j=1, \ldots, N$ ), evaluated in the monomorphic state when all individuals in the population play strategy $x$ (Appendix SB). The expression in equation (8) is the usual selection gradient on a mutant strategy in the island model (Rousset 2004, chapter 7); that is Hamilton's (1964) inclusive fitness effect. The first term represents the (direct) effect on a focal individual's personal fitness from an infinitesimal change of its own strategy, whereas the second term represents the (indirect) effect on the same individual's personal fitness from an infinitesimal change of the strategy of all its $N-1$ patch neighbors, weighted by pairwise relatedness in the neutral process. Uninvadability requires that the inclusive fitness effect be nil.

It turns out that the inclusive fitness effect must also be nil (eq. 8 must hold, Appendix SB) for a strategy $x$ to be a symmetric Nash equilibrium strategy under any of the two goal functions, $u_{\mathrm{A}}$ or $u_{\mathrm{B}}$. This suggests that there may be a link between maximizing behavior and uninvadability, but not how strong the link is. Suppose we have found a unique strategy that meets this first-order condition and that is also uninvadable. Then, we still do not know if this strategy is a Nash equilibrium strategy in the population game $G_{\mathrm{A}}=\left(\mathbb{N}, X, u_{\mathrm{A}}\right)$ or $G_{\mathrm{B}}=\left(\mathbb{N}, X, u_{\mathrm{B}}\right)$. Indeed, for either game, equilibrium requires, among other things, that the secondorder condition be met, but this condition is not necessarily the same as that for univadability. Next, we develop a numerical example to show that these conditions may very well differ.

\section{COUNTEREXAMPLE}

To see that that there can be a mismatch between uninvadability and maximizing behavior, let us consider a simple example of pairwise interactions $(N=2)$ in which expected fecundity is linear-quadratic in the two players' strategies,

$$
f(x, y)=\varphi\left[1+\alpha x-\beta x y-\gamma x^{2}\right],
$$

for some (large) baseline fecundity $\varphi$ common to every individual in the population, and where $x$ is the strategy of the focal individual, $y$ that of its patch neighbor, and $\alpha, \beta, \gamma$ are parameters. This fecundity function $f$ can be thought of as special case of the Cournot duopoly model (e.g., Fudenberg and Tirole 1991). Assuming a Moran reproductive process with fecundity effects 

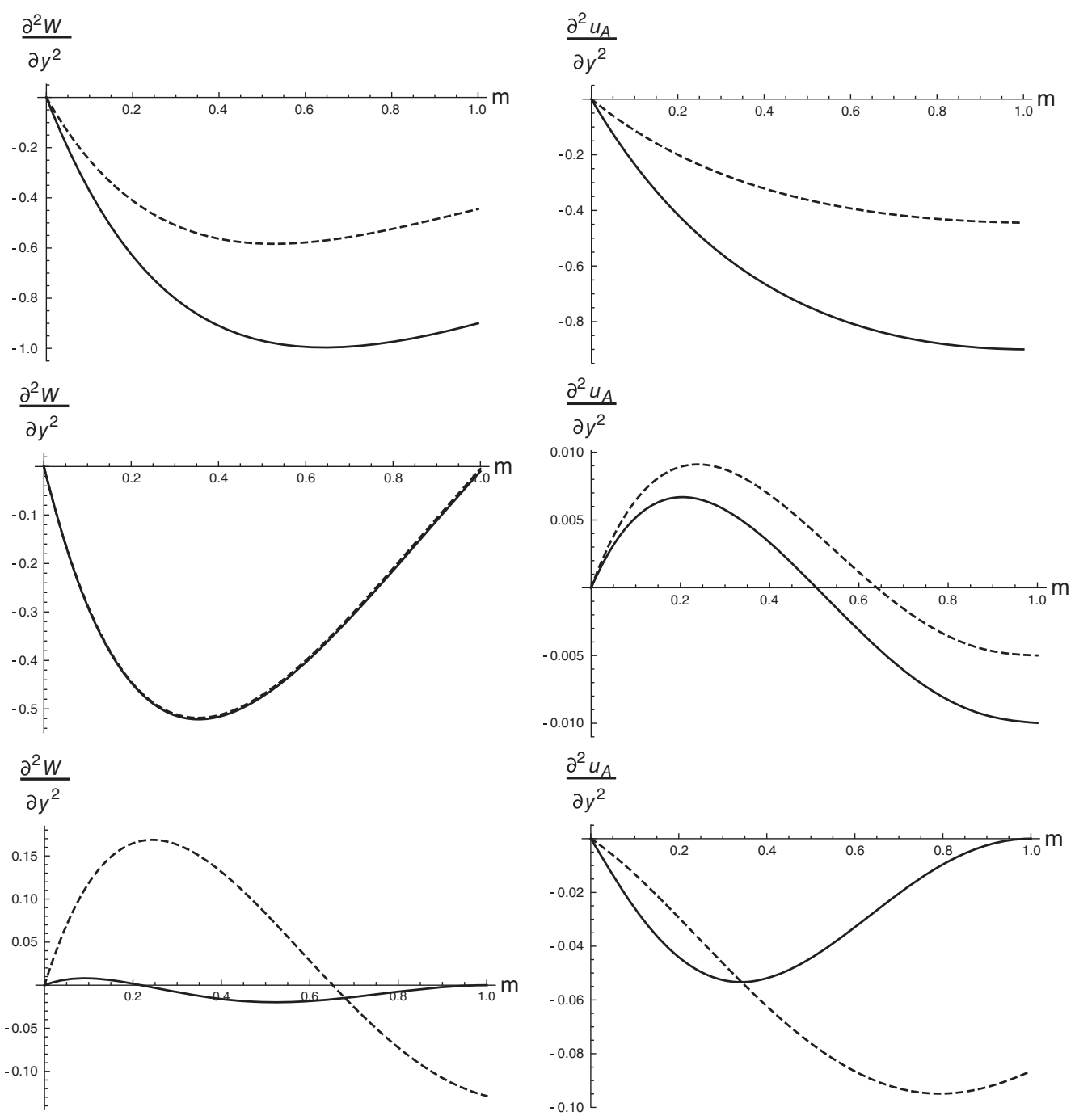

Figure 2. Graphs of the second-order derivatives given by equations (D-9) and (D-10) in Appendix SD as functions of migration $m$ evaluated at $x^{*}$ (eq. 10) for the Cournot game (eq. 9) under a Moran process when $N=2$. The first row of panels is for $\alpha=\beta=1$, and $\gamma=1(\gamma=0.5)$ for the plain (dashed) line. The second row of panels if for $\alpha=1, \beta=2$, and $\gamma=0.01(\gamma=0.005)$ for the plain (dashed) line. The third row of panels if for $\alpha=1, \beta=-1$, and $\gamma=0.5(\gamma=0.1)$ for the plain (dashed) line.

and without survival effects (Box 1, eq. B-a) and substituting fecundity (eq. 9) into personal fitness (eq. B-a) and then into the inclusive fitness effect (eq. 8) along with the relatedness for the Moran process (eq. B-e) shows that there is a unique element satisfying the first-order condition

$$
x^{*}=\frac{\alpha(3-m)}{2 \gamma(3-m)+2 \beta(2-m)} \text {. }
$$

For $\alpha=\beta=\gamma=1$, this strategy $x^{*}$ is uninvadable, $x^{*} \in$ $X_{\mathrm{U}}$, and is also a Nash equilibrium strategy with respect to goal function $u_{\mathrm{A}}, x^{*} \in X_{\mathrm{E}}\left(u_{\mathrm{A}}\right)$, whereby $X_{\mathrm{E}}\left(u_{\mathrm{A}}\right)=X_{\mathrm{U}}$ (see Fig. 2). In other words, maximizing behavior under $u_{\mathrm{A}}$ is equivalent with uninvadability for these parameter values. Suppose now that $\alpha=$ $1, \beta=2$, and $\gamma=0.01$. Then it is still true that $x^{*}$ is uninvadable, $x^{*} \in X_{\mathrm{U}}$. However, for low values of $m>0, x^{*}$ is no longer a
Nash equilibrium strategy (Fig. 2). One can then find a threshold value for $m \in(0,1)$, above which $X_{\mathrm{E}}\left(u_{\mathrm{A}}\right)=X_{\mathrm{U}}$ and below which $x^{*} \notin X_{\mathrm{E}}\left(u_{\mathrm{A}}\right)$ (in which case $X_{\mathrm{E}}\left(u_{\mathrm{A}}\right)=\varnothing$ ). Thus, maximizing behavior with respect to the $u_{\mathrm{A}}$ goal function is not equivalent to behavior that is uninvadable when $m$ is small.

Conversely, a strategy may be a Nash equilibrium strategy without being uninvadable. To see this, consider the case $\alpha=1$, $\beta=-1$, and $\gamma=0.5$. Then $x^{*}$ is a Nash equilibrium strategy, but for low values of $m$ (low migration rates) it is not uninvadable (Fig. 2). One can then find a threshold value (Appendix SB) for $m \in(0,1)$, above which $X_{\mathrm{E}}\left(u_{\mathrm{A}}\right)=X_{\mathrm{U}}$ and below which $x^{*} \notin X_{\mathrm{U}}$ (in which case $X_{\mathrm{U}}=\varnothing$ ). Thus, again, maximizing behavior with respect to the goal function $u_{\mathrm{A}}$ is not equivalent to behavior that is uninvadable when $m$ is small, that is, when dispersal is limited, which entails that identity-by-descent among group members is 
strong and kin selection is important $\left(q_{k} \gg 0, r \gg 0\right)$. Then, if fitness depends nonlinearly on the strategies, relatedness alone cannot describe the exact pattern of genealogical relationship between carriers of the strategies within patches (as it is only a summary statistic), and a comparison of the $u_{\mathrm{A}}$ goal function to lineage fitness (compare eqs. 3 and 6) reveals that we should expect a mismatch between the outcome of maximization of these two functions. Moreover, this mismatch is likely to be less serious for the $u_{\mathrm{B}}$ goal function as it will occur only if selection affects the genealogical relationship between individuals (compare eqs. 3 and 7). This intuition is confirmed by the results that we present next.

\section{TWO POSITIVE RESULTS}

We now characterize the conditions for the mismatch between maximizing behavior and uninvadability for the $u_{\mathrm{A}}$ and $u_{\mathrm{B}}$ goal function, respectively, in the case when the first-order condition has a unique solution. To that aim, it will be useful to introduce the following definitions. The strategies are (local) strategic complements, strategically neutral, or strategic substitutes in terms of personal fitness at $x$ if, respectively, they mutually reinforce each other's fitness effects, they have no impact on each other's fitness effects, or they weaken each other's fitness effects (formally, if $w_{i j}\left(x, \boldsymbol{x}_{x}, 1_{x}\right)$ is positive, zero, or negative for all $i, j \in\{1, . ., N\}$ with $i \neq j$, where $w_{i j}$ is the second-order partial derivative of $w\left(x_{i}, \boldsymbol{x}_{-i}, \phi\right)$ with respect to its $i$ th and $j$ th arguments, evaluated at a monomorphic resident $x$ ). Finally, we say that a strategy is (locally) relatedness increasing, relatedness neutral, or relatedness decreasing if it has a positive, zero, or negative effect on relatedness (formally if the derivative $r_{1}(x, x)=\partial r(y, x) /\left.\partial y\right|_{y=x}$ of relatedness with respect to its first argument is positive, zero, or negative).

With these definitions we can then establish sufficient conditions, in terms of the strategic character of the interaction and the relatedness effect of strategies for the following relations between uninvadability and maximizing behavior to obtain: (1) $X_{\mathrm{U}} \subseteq X_{\mathrm{E}}\left(u_{\mathrm{A}}\right)$, (2) $X_{\mathrm{E}}\left(u_{\mathrm{A}}\right) \subseteq X_{\mathrm{U}}$, and (3) $X_{\mathrm{E}}\left(u_{\mathrm{A}}\right)=X_{\mathrm{U}}$ (see Box 3 and proof Appendix SB). Let us consider the case when $x$ is uninvadable. In case (1), $x$ is also an equilibrium strategy in the game $G_{\mathrm{A}}$. In other words, an outside observer may interpret the behavior of the individuals in the population as maximizing with respect to the goal function $u_{\mathrm{A}}$. In case (2), either $x$ is an equilibrium strategy in $G_{\mathrm{A}}$ or else this game has no equilibrium strategy. In other words, an outsider who knows that $G_{\mathrm{A}}$ has at least one Nash equilibrium may again interpret the evolutionarily selected behavior of the individuals in the population as maximizing behavior with respect to the goal function $u_{\mathrm{A}}$. By contrast, if $G_{\mathrm{A}}$ has no equilibrium, then the outsider observing a strategy that is uninvadable cannot interpret it as being the outcome of maximizing $u_{\mathrm{A}}$. Finally, case (3) obtains when the strategies are relatedness neutral and strategically neutral. In particular, uninvadability is the same thing as maximizing behavior under the goal function $u_{\mathrm{A}}$ in pure decision problems, interactions in which personal fitness depends only on the individual's own action (then strategies are strategically neutral).

Box 3. Relation between maximizing behavior and unvadability. The forthcoming conditions apply when there is only a single strategy $x$ that satisfies the joint first-order condition of maximizing behavior and unvadability (eq. 8).

\section{Goal function $u_{\mathrm{A}}$ :}

(1) If $r_{1}(x, x) w_{N}\left(x, \boldsymbol{x}_{x}, 1_{x}\right) 0$ and the strategies are strategic complements at $x$ in terms of fitness, then $X_{\mathrm{U}} \subseteq X_{\mathrm{E}}\left(u_{\mathrm{A}}\right)$.

(2) If $r_{1}(x, x) w_{N}\left(x, \boldsymbol{x}_{x}, 1_{x}\right) \leq 0$ and the strategies are strategic substitutes at $x$ in terms of fitness, or $r_{1}(x, x)$ $w_{N}\left(x, \boldsymbol{x}_{x}, 1_{x}\right) \geq 0$ and the strategies are strategically neutral at $x$ in terms of fitness, then $X_{\mathrm{E}}\left(u_{\mathrm{A}}\right) \subseteq X_{\mathrm{U}}$.

(3) If $r_{1}(x, x) w_{N}\left(x, \boldsymbol{x}_{x}, 1_{x}\right)=0$ and the strategies are strategically neutral at $x$ in terms of fitness, then $X_{\mathrm{E}}\left(u_{\mathrm{A}}\right)=X_{\mathrm{U}}$.

\section{Goal function $u_{\mathrm{B}}$ :}

(1) If $r_{1}(x, x) w_{N}\left(x, \boldsymbol{x}_{x}, 1_{x}\right)>0$, then $X_{\mathrm{U}} \subseteq X_{\mathrm{E}}\left(u_{\mathrm{B}}\right)$.

(2) If $r_{1}(x, x) w_{N}\left(x, \boldsymbol{x}_{x}, 1_{x}\right)<0$, then $X_{\mathrm{E}}\left(u_{\mathrm{B}}\right) \subseteq X_{\mathrm{U}}$.

(3) If $r_{1}(x, x) w_{N}\left(x, \boldsymbol{x}_{x}, 1_{x}\right)=0$, then $X_{\mathrm{E}}\left(u_{\mathrm{B}}\right)=X_{\mathrm{U}}$.

By contrast, the goal function $u_{\mathrm{B}}$ has a closer tie than $u_{\mathrm{A}}$ to uninvadability as the sufficient conditions for the relations between uninvadability and maximizing behavior to hold can be expressed solely in terms of the relatedness effect of strategies, and are thus less demanding (see Box 3). Comparing the conditions for $u_{\mathrm{A}}$ and $u_{\mathrm{B}}$ in Box 3 , one sees that an uninvadable strategy can be an equilibrium strategy in game $G_{\mathrm{B}}$ without being an equilibrium strategy in game $G_{\mathrm{A}}$. By contrast, an uninvadable strategy cannot be an equilibrium strategy in $G_{\mathrm{A}}$ without also being an equilibrium strategy in $G_{\mathrm{B}}$. Similarly, there are situations in which an equilibrium strategy in game $G_{\mathrm{B}}$ is also uninvadable without being an equilibrium strategy in the game $G_{\mathrm{A}}$, whereas the reverse case cannot arise.

The link between natural selection, as expressed by uninvadability, and "as if" maximizing behavior is hence in general stronger for the goal function $u_{\mathrm{B}}$ than for the goal function $u_{\mathrm{A}}$. Moreover, it is sufficient that strategies are relatedness neutral for evolution to result in the same behaviors "as if" maximization under goal function $u_{\mathrm{B}}$. Fo instance, in the above example (eq. 9) under a Moran process, maximizing behavior with respect to the $u_{\mathrm{B}}$ goal function is equivalent to uninvadability because $x^{*}$ (eq. 10) is relatedness neutral. 
Finally, note that if $m=1$ (the population is panmictic), then $q_{k}\left(\theta^{\prime}, \theta\right)=1$ for $k=1$ (hence zero for all other $k$ ), and $r(x, x)=0$, in which case $u_{\mathrm{A}}$ and $u_{\mathrm{B}}$ both reduce to $w\left(x_{i}, \boldsymbol{x}_{-i}, \phi\right)$. Maximizing personal fitness is then equivalent to maximizing lineage fitness (eq. 3). We conclude that in a panmictic population the correspondence between maximizing behavior and uninvadability obtains $\left(X_{\mathrm{E}}\left(u_{\mathrm{B}}\right)=X_{\mathrm{E}}\left(u_{\mathrm{A}}\right)=X_{\mathrm{U}}\right)$, and this holds for general strategy spaces.

\section{Weak Selection UNINVADABILITY}

We now turn to the study of uninvadability under weak selection and assume that types can only affect fecundity or survival, but not both simultaneously and also do not affect individuals' migration rate, which henceforth is constant. Suppose that types affect only fecundity and assume that the expected fecundity of any individual $i$ can be written under the form

$$
f\left(\theta_{i}, \boldsymbol{\theta}_{-i}, \phi\right)=\varphi\left[1+\delta \pi\left(\theta_{i}, \boldsymbol{\theta}_{-i}, \phi\right)\right],
$$

where $\pi$ can be thought of as the expected material payoff obtained during the stage of social interactions (stage (1) of the life cycle). The parameter $\delta \geq 0$ represents the intensity of selection. If $\delta=0$, then every individual has exactly the same fecundity and hence same fitness, which entails that the evolutionary process is neutral. The assumption behind equation (11) is that fecundity can be expressed in terms of material payoff such that the outcome of the interaction affects reproduction only weakly, which can be justified by noting that fitness can depend on many other phenotypes, such as morphology and physiology (which under the time span considered are taken to be fixed in the population).

If the type $\theta_{i}$ affects the survival of an adult individual $i$ (e.g., eq. B-b), then a positive relationship between material payoff and individual survival, in the same vein as in equation (11), can be postulated. Irrespective of specification, most standard models of evolutionary population dynamics (e.g., Frank 1998; Ewens 2004; Rousset 2004 and eq. B-b) exhibit the following three canonical properties: (1) personal fitness is increasing in the fecundity (or survival) of the individual, and therefore also in the individual's material payoff $\pi\left(\theta_{i}, \boldsymbol{\theta}_{-i}, \phi\right)$; (2) personal fitness is decreasing in the fecundity (or survival) of the individual's patch neighbors, and therefore the fitness of an individual $i$ is decreasing in the material payoff $\pi\left(\theta_{j}, \boldsymbol{\theta}_{-j}, \phi\right)$ to a patch neighbor $j \neq i$; (3) an individual's personal fitness is more sensitive to changes in its own fecundity (or survival) than to changes in any (individual) neighbor's fecundity (or survival). In the subsequent analysis, we will focus on the class of evolutionary dynamics under which fitness functions exhibit these qualitative properties.
We show in Appendix SC that under these assumptions, and for $\delta$ close to zero, the lineage fitness to type $\theta^{\prime} \in\{\theta, \tau\}$ can be expressed in terms of the lineage payoff to type $\theta^{\prime}$ in a resident $\theta$ population,

$$
\Pi\left(\theta^{\prime}, \theta\right)=\sum_{k=1}^{N} \sum_{\theta_{-i} \in S_{k}\left(\theta^{\prime}, \theta\right)} \pi_{\mathrm{R}}\left(\theta^{\prime}, \boldsymbol{\theta}_{-i}, 1_{\theta}\right) q_{k}^{\circ} .
$$

Here, $q_{k}^{\circ}$ is the profile distribution evaluated at $\delta=0$, which entails that the evolutionary process is neutral and independent of types, and

$$
\pi_{\mathrm{R}}\left(\theta_{i}, \boldsymbol{\theta}_{-i}, 1_{\theta}\right)=\pi\left(\theta_{i}, \boldsymbol{\theta}_{-i}, 1_{\theta}\right)-\frac{\lambda}{N-1} \sum_{j \neq i} \pi\left(\theta_{j}, \boldsymbol{\theta}_{-j}, 1_{\theta}\right)
$$

is the payoff advantage to a focal individual $i$ over its patch neighbors. The parameter $\lambda$ is "the spatial scale of density-dependent competition" (Frank 1998, p. 115), a parameter that takes values between zero and one and quantifies the intensity of local competition between patch members for breeding spots. An increase in the average material payoff to the focal individual's patch neighbors by a small amount $\delta$ would increase the local density-dependent competition experienced by the focal individual (during stage [4] of its life cycle) by $\delta \lambda$. Hence, if $\lambda=0$, the lineage payoff to any type $\theta^{\prime} \in\{\theta, \tau\}$ in a resident $\theta$ population is simply the material payoff to a carrier of type $\theta^{\prime}$ in such a population. By contrast, if $\lambda=1$, then the lineage payoff is the material payoff of carriers of type $\theta^{\prime}$ over patch neighbors (see Appendix SC for examples of how $\lambda$ values can be derived from demographic processes). As such, the parameter $\lambda$ captures any "spite" effect due to the finite number of individuals within patches (e.g., Gardner and West 2004).

We will call a type $\theta \in \Theta$ uninvadable under weak selection if it solves the maximization problem (4) when $\delta$ goes to zero. One can show that this is equivalent with maximization of lineage payoff, namely solving the maximization problem

$$
\max _{\tau \in \Theta} \Pi(\tau, \theta)
$$

The fact that attention may be restricted to only material payoffs is a consequence of the assumptions that fitness increases (approximately) linearly with material payoff (for small $\delta>0$ ) and that the migration probability is the same for all individuals. Indeed, under the latter assumption, fitness depends on individuals' strategies only through material payoffs. Second, the fact that the probability weights in equation (12) are given by the neutral population process is again a consequence of weak selection; indeed, in the limit when $\delta$ goes to zero, fitness is the same for everyone. 


\section{MAXIMIZING BEHAVIOR}

We can now turn to the "as if" question under weak selection. In contrast to the analysis under strong selection, here we do not make any structural assumption about the set $X$, which may be of arbitrarily dimension. We consider the goal function $u_{\mathrm{C}}$ defined by

$$
u_{\mathrm{C}}\left(x_{i}, \boldsymbol{x}_{-i}, \phi\right)=\sum_{k=1}^{N} \sum_{\tilde{\boldsymbol{x}}_{-i} \in P_{k}\left(\boldsymbol{x}_{-i}\right)} \pi_{\mathrm{R}}\left(x_{i}, \tilde{\boldsymbol{x}}_{-i}, \phi\right) q_{k}^{\circ} .
$$

This goal function represents the individual's expected payoff advantage over its patch neighbors, where the expectation is taken with respect to the local lineage distribution in the neutral process.

Let $X_{\text {Uw }} \subseteq X$ denote the set of uninvadable strategies under weak selection. Uninvadability under weak selection coincides exactly with maximizing behavior with respect to the $u_{\mathrm{C}}$ goal function, that is,

$$
X_{\mathrm{E}}\left(u_{\mathrm{C}}\right)=X_{\mathrm{UW}}
$$

In sum, in a model with no assumptions on the specifics of the nature of the social interaction (one-shot or multistage, observed or unobserved actions of patch members, etc.), we find that under weak selection evolution selects behaviors that are identical with those that emerge if individuals strive to maximize $u_{\mathrm{C}}$. This is our main positive result (see Appendix SC for a proof).

\section{EXAMPLE}

When $N=2$, the goal function (eq. 15) for an individual $i$ who plays $x_{i}=x$, whereas its patch neighbor plays $x_{-i}=y$ can be written as

$$
\begin{aligned}
u_{\mathrm{C}}(x, y, \phi)= & (1-\lambda)(1-r) \pi(x, y, \phi)+(1-\lambda) r \pi(x, x, \phi) \\
& +\lambda(1-r)[\pi(x, y, \phi)-\pi(y, x, \phi)] .
\end{aligned}
$$

This is a weighted sum of three terms, where the weight $(1-\lambda)(1-r)$ is given to one's own payoff, the weight $(1-\lambda) r$ to the payoff that each individual would obtain had the other individual used the same strategy, and the weight $\lambda(1-r)$ is given to the individual's payoff advantage.

For a Moran process (Box 1), we have $r=(1-m) /(1+m)$ (this follows from eq. B-e) and $\lambda=(1-m)^{2} /\left[2-(1-m)^{2}\right]$ (see Appendix SA). Inserting this along with the material payoff function $\pi(x, y, \phi)=\alpha x-\beta x y-\gamma x^{2}$ (which captures the same social interaction as eq. 9) into equation (17), we obtain that the first-order condition $\partial u_{\mathrm{C}}(x, y, \phi) /\left.\partial x\right|_{y=x}=0$ has a unique solution given by equation (10). Hence, if this strategy also satisfies the second-order condition $\partial^{2} u_{\mathrm{C}}(x, y, \phi) /\left.\partial x^{2}\right|_{y=x}<0$, then this strategy is the unique symmetric Nash equilibrium strategy in the population game $G_{\mathrm{C}}=\left(\mathbb{N}, X, u_{\mathrm{C}}\right)$. By the result given in equation (16), this strategy is then also the unique uninvadable strategy. In this example, the second-order condition boils down to $(1-m)(2-m) \beta+(3-m) \gamma>0$.

\section{Discussion}

We have examined whether, in a patch-structured population, a strategy that is uninvadable can be interpreted as being freely chosen by individuals who seek to maximize some individualcentered goal function. For the purpose of analyzing this "as if" question, we have examined individual-centered goal functions that are expressed in terms of personal fitness or personal material payoff. These candidate functions are analytically operational and also transparent in the sense that they explicitly depend on the fitnesses of, or material payoffs to, the individual and its partners engaged in the social interaction at hand, weighted by certain "population-structural" coefficients exogenous to the individual.

Our results can be summarized as follows:

(1) Arbitrary selection strength. We studied two individualcentered goal functions, one in line with the textbook representation of inclusive fitness $\left(u_{\mathrm{A}}\right.$, eq. 6$)$ and one $\left(u_{\mathrm{B}}\right.$, eq. 7$)$ that is closer to a population-statistical version of inclusive fitness. It turns out that neither goal function gives rise to "as if" behavior in general although the population-statistical version fares better (results Box 3). An important exception is the case of strategically neutral interactions, for which both goal functions $\left(u_{\mathrm{A}}\right.$ and $\left.u_{\mathrm{B}}\right)$ fare equally well.

(2) Weak selection. We studied a third individual-centered goal function $\left(u_{\mathrm{C}}\right.$, eq. 15$)$ that is a certain weighted average of material payoffs to oneself and one's patch neighbors. This goal function gives rise to "as if" behavior regardless of the complexity and strategic nature of the social interaction.

We will now discuss more in detail the scope and interpretation of these results.

\section{PATCHES, FAMILIES, AND PANMICTIC POPULATIONS}

Our analysis shows that, even when a concept of invasion-fitness maximization applies under strong selection (lineage fitness in our formalization), the "as if" notion of an individual as an agent maximizing a goal function with population-structure coefficients exogenous to its own behavior does not necessarily obtain. This stems from the fact that lineage fitness generally depends on the full distribution of types that a carrier of the mutant trait is exposed to, and this distribution in turn depends on the expression of the mutant and resident type in past generations. Hence, lineage fitness is a complex multigenerational measure of invasion fitness, where the distribution of types is endogenously determined and thus depends on selection. Because this dependency is endogenous by nature, a goal function representing 
lineage fitness cannot in general be written as a linear combination of personal fitness functions with population-structure coefficients that are independent of the fitness of the different types and thus of selection.

This argument applies regardless of the dimensionality of the type space. Hence, our results (Box 3) establish the fact that, in general, no full correspondence exists between the set of Nash equilibria induced by maximizing behavior under conventional goal functions and the set of uninvadable strategies. But there are exceptions to these negative results. For instance, in evolutionary biology and evolutionary game theory the canonical model of social interactions is symmetric pairwise interactions in a panmictic population (e.g., Maynard Smith 1982; Eshel 1983; Weibull 1997), which, by definition, is a situation where there is no local competition and no relatedness. In this case, lineage fitness is proportional to personal fitness, which in turn is an affine increasing function of material payoff. Then, an individual-centered goal function that produces "as if maximizing" behavior can be readily found and is directly given by the material-payoff function of the social interaction, which implies that uninvadability is equivalent to equilibrium play in the social interaction. Our model allows for a direct extension of this case to multiplayer interactions within groups of any size in a panmictic population, a case that has been extensively studied in evolutionary biology (e.g., the "haystack" model, Maynard Smith 1964, or the "groupselection" and "founder effect" models, Wilson 1975; Cohen and Eshel 1976). This is a general result, regardless of the game being played between group members.

Other general cases of maximizing behavior can be found by considering pairwise interactions among family members in a panmictic population, for instance interactions among siblings (or parent-offspring interactions) before the round of complete dispersal, where the kinship structure is determined in a single episode of reproduction and does not depend on the type distribution when mutants are rare. For this case, invasion fitness is proportional to a convex combination of (1) a mutant's material payoff when matched with another mutant and (2) a mutant's material payoff when matched with a resident, with a constant weight $r$ placed on the first payoff. Namely, $W(y, x) \propto r \pi(y, y)+(1-r) \pi(y, x)$, where $r$ is pairwise relatedness (Day and Taylor 1998), which does not depend on the types. In this case, uninvadability is equivalent with maximizing behavior under the individual-centered goal function $u_{\mathrm{F}}(y, x)=$ $r \pi(y, y)+(1-r) \pi(y, x)$ because $X_{\mathrm{E}}\left(u_{\mathrm{F}}\right)=X_{\mathrm{U}}$. This goal function was used in Bergstrom (1995) for interactions between full siblings and analyzed more generally in Alger and Weibull (2013), and it is equivalent to the $u_{\mathrm{C}}$ goal function in the absence of rivalry and when the material payoff does not depend on the behavior of individuals in other patches (eq. 17). Hence, in familystructured populations maximizing behavior can obtain for all games under the condition that relatedness is not affected by selection.

More generally, as discussed above, what renders a general "as if maximizing" representation unfeasible when populationstructure coefficients are exogenous to the individual, is the fact that a population's genetic structure generally depends on selection. It would thus be useful for future research to delineate the instances of family (or spatially) structured populations where invasion fitness depends on population-structural coefficients that are independent of selection or only weakly affected by it.

\section{WEAK SELECTION}

Under weak selection, all earlier events of selection can be summarized by a neutral distribution of types independent of the mutant, and which quantifies the effects of the kinship structure induced by limited dispersal on an individual's goal function (the distribution $q_{k}$ is independent of the mutant, see eq. 12). Hence, an individual-centered goal function, with populationstructural coefficients independent of selection, can be found. We showed that, regardless of the complexity of social interactions, individuals who maximize their average payoff advantage would choose, in equilibrium, strategies that are uninvadable. We note that this result is nevertheless not fully general as it applies only to traits affecting survival or reproduction, but not migration rates or other traits modifying the genetic system (i.e., modifier traits).

Although we rule out modifier traits by assumption, we impose virtually no restrictions on the games individuals play. Our weak-selection result covers maximizing behavior for games where strategies are strategic substitutes or complements, and the special case of strategically neutral games. A special case of strategically neutral games are games in which the payoff function is additively separable in the strategies used by different group members. In such games, a goal function that takes the same form as the $u_{\mathrm{A}}$ goal function (eq. 6), but where fitness is replaced by material payoff, would also produce "as if maximizing" behavior. This is analogous to the situation considered in Grafen (2006), so our results match his results about "optimization of inclusive fitness" with constant environmental states. Strategically neutral games can be viewed as independent decision problems, one for each player, and so the concept of Nash equilibrium to characterize maximizing behavior is not needed in this special situation. This is probably the reason why this fundamental concept does not appear previously in the literature in evolutionary biology on maximizing behavior in the context of interactions between relatives (e.g., Grafen 2006; Gardner and Welch 2011), a literature that usually deals only with strategically neutral games. This previous work also endorses a concept of uninvadability of a resident type evaluated from the action of natural selection over only a single demographic time period, the initial period where 
the mutant arises (e.g., Grafen 2006, p. 553), and which is in general dynamically insufficient to ascertain the stability of a resident type when interactions occur between relatives (Lehmann and Rousset 2014). By contrast, uninvadability in our analysis is ascertained from a multigenerational measure of invasion fitness (lineage fitness), which is consistent with standard evolutionary analysis (Ferrière and Gatto 1995; Metz and Gyllenberg 2001; Rousset 2004).

Finally, it is worth noting that because different goal functions can produce the same behavior, the goal functions introduced in the "as if" approach are not uniquely defined. For instance, any strictly increasing transformation of a given goal function returns a new goal function with the same set of maximands. Nevertheless, the goal function producing "as if" behavior that we have identified ( $u_{\mathrm{C}}$, see eq. 15) combines three components that are likely to appear generally (see also eq. 17). These are (1) a "self-interest" component (one's own material payoff), which ultimately results from fitness depending on the individual's own material payoff; (2) a "group-interest" component (group material payoff that would arise if others behaved like oneself), whereby individuals can be thought as evaluating the consequences of their behaviors on average group material payoff by assuming that others would choose the same behavior, and which ultimately results from identity-by-descent within patches and cause individuals to express identical strategies; and, (3) a "competitiveness" or "rivalry" component (material payoff differences), which results from local competition in a spatially structured population, which makes the fitness of an individual decrease in the others' material payoffs.

These last two components are direct consequences of limited dispersal. They are the goal-oriented behavioral consequences of kin-selected benefits (or costs) and kin competition, the two fundamental and general additional components of the selection pressure on any social behavior induced by limited dispersal (e.g., Grafen 1984; Queller 1994; West et al. 2002; Rousset 2004). Note that the "group-interest" component of the goal function $u_{\mathrm{C}}$, can be thought of as representing "Kantian morality" (see Alger and Weibull 2013, section 6.2 for a discussion of this). We also conjecture that the three qualitative components of the $u_{\mathrm{C}}$ goal function will also emerge under more realistic demographics, such as class or demographically structured populations, something that could be detailed in future work. Finally, we note that if we follow Dawkins (1978, p. 63) and define inclusive fitness as "that property of an individual organism which will appear to be maximized when what is really being maximized is gene survival," then the $u_{\mathrm{C}}$ goal function can be thought of as a representation of inclusive fitness.

\section{CONSTRAINED BEHAVIOR}

We formulated the relationship between maximizing behavior and uninvadability in terms of individuals (freely) choosing their strategies, where a strategy is a complete plan of action for all possible contingencies. As is well-known in game theory (see, e.g., van Damme 1987), a (behavior) strategy profile is a Nash equilibrium if and only if it prescribes optimal continuation play from every information set that is reached with positive probability (while actions, or continuation play, at unreached information sets need not be optimal).

Consider a social interaction in which each participant has a behavior rule that implements a behavior strategy for that individual. A situation in which each action in the continuation play from any information set onwards can evolve to optimality entails that the set $\mathcal{S}$ of states of an individual is very large. This has been used in models without social interactions in behavioral ecology (i.e., decision problems where individuals interact with their exogenous environment, e.g., McNamara and Houston 1999). But it is rarely (if ever) considered in biological models of social interactions, as the set $\mathcal{S}$ of states is usually taken to be of small dimension, a modeling choice often following from the observation that most animals (including human) decision making is cognitively bounded (Fawcett et al. 2012). A low-dimensional state space cannot represent the whole history of actions $\mathcal{H}$, and the behavior rule itself is further usually assumed to depend only on a low-dimensional evolvable type. A low-dimensional behavior rule can thus fundamentally constrain the flexibility of behavior in social interactions, and this curtails the possibility of actions that are all optimal along the path of play.

The interpretation of maximizing behavior can accommodate such mechanistic constraints by assuming that individuals choose the parameters (or variables) of the behavior rule among the given set of alternatives (in Appendix SC we develop a model illustrating these concepts where individuals play a repeated game but have a memory of step one). In other words, the parameters determining the mechanism that generates actions (the behavior rule) are optimal for each individual, in terms of the goal function $u_{\mathrm{C}}$, when these parameters take the values that are uninvadable, and can thus be interpreted as being the results of maximizing behavior. This reasoning applies to any trait affecting any behavior rule, such as traits affecting cognitive properties such as memory size, learning speed, or internal reward systems involved in decision making. In sum, the interpretation in terms of maximizing behavior can be applied to both flexible and constrained behavior, and any evolvable phenotype determining a proximate mechanism that generates actions can be interpreted as a strategy in a corresponding game. 


\section{EVOLUTIONARY SELECTION OF GOAL FUNCTIONS}

Our "as if" question led us to posit three alternative individualcentered goal functions and to compare the strategies that would result from maximization of these goal functions to those being selected for by way of natural selection. However, under environmental variability, selection may act directly at the level of goal functions in cognitively sophisticated organism, and so to speak delegate the (free) choice of action(s) to the individual. One may then ask which goal functions will be selected for (hence the goal function itself becomes an evolving "strategy"). This question is distinct from the "as if" question addressed here and has been analyzed previously by economists (Alger and Weibull 2012, 2013, and references therein) and biologists (Akçay et al. 2009; Akçay and Van Cleve 2012). The present modeling framework could be applied to such an analysis, in which case each type would define a goal function, and the set of types would be all the goal functions that the organism's cognition and physiology could implement. Importantly, as suggested by the contrasting results in Alger and Weibull (2012) and Alger and Weibull (2013), in such an analysis it will matter if organisms can recognize each others' type or not. We conjecture (by extrapolating from the analysis in Alger and Weibull 2013 and our results here) that when evolution occurs at the level of goal functions and organisms cannot recognize each others' type, the $u_{\mathrm{C}}$ goal function (eq. 15) is uninvadable under weak selection. Under strong selection, however, all caveats about the correspondence between uninvadability and maximizing behavior discussed above may be expected to apply to the evolution of goal functions when organisms cannot recognize each other's type.

\section{Conclusion}

Because in our model there are no genetic constraints, evolvable traits can be thought of as being coded by a one-locus genetic basis. Our model thus provides a framework in which the conditions are ideal for identifying maximizing behavior under evolutionary dynamics. Within this framework we show that when social interactions are modeled as games between population members, there is only a partial correspondence between gene-centered maximization and conventional individual-centered maximization, unless selection is weak. But individuals can still be the instruments of the gene's goal, and our results are consistent with the view that this is the level at which adaptation and thus maximizing behavior can be conceived in complete generality (Dawkins 1978; Haig 2012).

\section{ACKNOWLEDGMENTS}

We thank C. Mullon for useful discussions on various steps taken in the Appendix. We also thank C. Clavien and A. Grafen for useful discussions and are grateful to a reviewer for helping us increase the intelligibility of the paper. This work is partly supported by French National Research Agency (ANR) grant Chaire d'Excellence to IA, Swiss National Science Foundation grant PP00P3-146340 to LL, and French ANR-11-IDEX-0002-02 to JW.

\section{LITERATURE CITED}

Ajar, E. 2003. Analysis of disruptive selection in subdivided populations. BMC Evol. Biol. 3:1-12.

Akçay, E., and J. Van Cleve. 2012. Behavioral responses in structured populations pave the way to group optimality. Am. Nat. 179:257-269.

Akçay, E., J. Van Cleve, M. W. Feldman, and J. Roughgarden. 2009. A theory for the evolution of other-regarding motivations integrating proximate and ultimate perspectives. Proc. Natl. Acad. Sci. USA 106:1906119066.

Alcock, J. 2005. Animal behavior: an evolutionary approach. Sinauer Associates, Cambridge, MA.

Alger, I., and J. W. Weibull. 2012. A generalization of Hamilton's rule-love others how much? J. Theor. Biol. 299:42-54.

- 2013. Homo moralis: preference evolution under incomplete information and assortative matching. Econometrica 81:2269-2302.

Bergstrom, T. 1995. On the evolution of altruistic ethical rules for siblings. Am. Econ. Rev. 85:58-81.

Binmore, K., 2011. Rational decisions. Princeton University Press, Princeton, NJ.

Caswell, H. 2000. Matrix population models. Sinauer Associates, Cambridge, MA.

Chesson, P. L. 1981. Models for spatially distributed populations: the effect of within-patch variability. Theor. Popul. Biol. 19:288-325.

Clobert, J., E. Danchin, A. A. Dhondt, and J. D. Nichols, eds. 2001. Dispersal. Oxford University Press, Oxford.

Cohen, D., and I. Eshel. 1976. On the founder effect and the evolution of altruistic traits. Theor. Popul. Biol. 10:276-302.

Crow, J. F. and M. Kimura. 1970. An introduction to population genetics theory. Harper and Row, New York.

Dawkins, R. 1978. Replicator selection and the extended phenotype. Zeitschrift für Tierpsychologie 47:61-76.

1982. The extended phenotype. Oxford University Press, Oxford, U.K.

Day, T., and P. Taylor. 1998. The evolution of temporal patterns of selfishness, altruism, and group cohesion. Am. Nat. 152:102-113.

Dekel, E., C. E. Jeffrey, and O. Yilankaya. 2007. Evolution of preferences. Rev. Econ. Stud. 74:685-704

Enquist, M., and S. Ghirlanda. 2005. Neural networks and animal behavior. Princeton University Press, Princeton, NJ.

Eshel, I. 1983. Evolutionary and continuous stability. J. Theor. Biol. 103:99111.

1991. Game theory and population dynamics in complex genetical systems: the role of sex in short term and in long term evolution. Pp. 6-28 in R. Selten, ed. Game equilibrium models I. Springer, Heidelberg.

- 1996. On the changing concept of evolutionary population stability as a reflection of a changing point of view in the quantitative theory of evolution. J. Math. Biol. 34:485-510.

Eshel, I., M. Feldman, and A. Bergman. 1998. Long-term evolution, short-term evolution, and population genetic theory. J. Theor. Biol. 191:391-396.

Ewens, W. J. 2004. Mathematical population genetics. Springer-Verlag, New York. 2011. What is the gene trying to do? Br. J. Philos. Sci. 62:155-176. 
Fawcett, T., S. Hamblin, and L. A. Giraldeau. 2012. Exposing the behavioral gambit: the evolution of learning and decision rules. Behav. Ecol.: 24:211.

Ferrière, R., and M. Gatto. 1995. Lyapunov exponents and the mathematics of invasion in oscillatory or chaotic populations. Theor. Popul. Biol. 48:126-171.

Fisher, R. A. 1930. The genetical theory of natural selection. Clarendon Press, Oxford, U.K.

Frank, S. A. 1998. Foundations of social evolution. Princeton University Press, Princeton, NJ.

Fudenberg, D., and J. Tirole. 1991. Game theory. MIT Press, Cambridge, MA.

Gardner, A., and J. J. Welch. 2011. A formal theory of the selfish gene. J. Evol. Biol. 24:1801-1813.

Gardner, A., and S. A. West. 2004. Spite and the scale of competition. J. Evol. Biol. 17:1195-1203.

Grafen, A. 1984. Natural selection, kin selection and group selection. Pp. 62-84 in J. R. Krebs and N. Davies, eds. Behavioural ecology: an evolutionary approach. Blackwell Scientific Publications, Oxford.

- 2006. Optimization of inclusive fitness. J. Theor. Biol. 238:541563.

Haig, D. 1997. The social gene. Pp. 284-304, in J. R. Krebs and N. Davies, eds. Behavioural ecology: an evolutionary approach. 4th ed. Chap. 12. Blackwell, Oxford, U.K.

2012. The strategic gene. Biol. Philos. 27:461-479.

Hamilton, W. D. 1964. The genetical evolution of social behaviour, 1. J. Theor. Biol. 7:1-16

Haykin, S. 1999. Neural networks: a comprehensive foundation. 2nd ed. Prentice Hall, Upper Saddle River.

Heifetz, A., C. Shannon, and S. Y. 2007. The dynamic evolution of preferences. Econ. Theory 32:251-286.

Kingman, J. 1961. A mathematical problem in population genetics. Math. Proc. Cambridge Philos. Soc. 57:574-582.

Kreps, D. M. 1988. Notes on the theory of choice. Westview Press, Denver.

Lehmann, L., and F. Rousset. 2014. Fitness, inclusive fitness, and optimization. Biol. Philos. 29:181-195.

Leimar, O. 1997. Repeated games: a state space approach. J. Theor. Biol. 184:471-498.

Maynard, Smith J. 1964. Group selection and kin selection. Nature 201:11451147.

- 1982. Evolution and the theory of games. Cambridge University Press, Cambridge, U.K.

Maynard, Smith, J., and G. R. Price. 1973. The logic of animal conflict. Nature 246:15-18.

McFarland, D., and A. Houston. 1981. Quantitative ethology: the state space approach. Pitman, Boston, MA.
McNamara, J. M., and A. I. Houston. 1999. Integrating function and mechanism. Trends Ecol. Evol. 24:670-675.

Metz, J., and M. Gyllenberg. 2001. How should we define fitness in structured metapopulation models? Including an application to the calculation of evolutionary stable dispersal strategies. Proc. R. Soc. Lond. B Biol. Sci. 268:499-508.

Metz, J. A. J. 2011. Thoughts on the geometry of meso-evolution: collecting mathematical elements for a post-modern synthesis Pp. 193-231. In F. A. C. C. Chalub and J. Rodrigues, eds. The mathematics of Darwin's legacy, mathematics and biosciences in interaction. Birkhäuser, Basel.

Minsky, M. L. 1967. Computation: finite and infinite machines. Prentice Hall, Upper Saddle River.

Moran, P. A. P. 1964. On the nonexistence of adaptive topographies. Ann. Hum. Genet. 27:383-393.

Mullon, C., and L. Lehmann. 2014. The robustness of the weak selection approximation for the evolution of altruism against strong selection. J. Evol. Biol. 27:2272-2282.

Nagylaki, T. 1992. Introduction to population genetics. Springer-Verlag, Heidelberg.

Osborne, J. M., and A. Rubinstein. 1994. A course in game theory. MIT Press, Cambridge, MA.

Queller, D. C. 1994. Genetic relatedness in viscous populations. Evol. Ecol. 8:70-73.

Rousset, F. 2004. Genetic structure and selection in subdivided populations. Princeton University Press, Princeton, NJ.

Roze, D., and F. Rousset. 2008. Multilocus models in the infinite island model of population structure. Theor. Popul. Biol. 73:529-542.

Taylor, P. 1990. Allele-frequency change in a class-structured population. Am. Nat. 135:95-106.

van Damme, E. 1987. Stability and perfection of Nash equilibria. SpringerVerlag, Berlin.

Weibull, J. W. 1997. Evolutionary game theory. MIT Press, Cambridge, MA.

West, S. A., I. Pen, and A. S. Griffin. 2002. Cooperation and competition between relatives. Science 296:72-75.

Wild, G. 2011. Inclusive fitness from multitype branching processes. Bull. Math. Biol. 73:1028-1051. Available at http://www.ncbi.nlm.nih.gov/ pubmed/20532644.

Wilson, D. S. 1975. A theory of group selection. Proc. Nat. Acad. Sci. USA 72:143-146.

Wright, S. 1931. Evolution in Mendelian populations. Genetics 16:97-159. - 1942. Statistical genetics and evolution. Bull. Am. Math. Soc. 48:223-246.

Associate Editor: J. Masel Handling Editor: M. Servedio

\section{Supporting Information}

Additional Supporting Information may be found in the online version of this article at the publisher's website:

Appendix A: Univadability.

Appendix B: Univadability and maximizing behavior.

Appendix C: Weak selection.

Appendix D: Moran process calculations.

Appendix E: Constrained behavior. 


\section{Supplementary material for "Does evolution lead to maximizing behavior?" by L. Lehmann, I. Alger, and J. Weibull}

\section{Appendix A: univadability}

We here prove that a mutant type $\tau$ appearing initially as a single copy on a single focal island of the population, which is otherwise fixed for the resident $\theta$, will go extinct with probability one if, and only if, $\theta$ solves the problem $\max _{\tau \in \Theta} W(\tau, \theta)$; that is, if, and only if, $W(\tau, \theta) \leq W(\theta, \theta)$ for all $\tau \in \Theta$. Our proof below follows the line of arguments developed in Mullon et al. (in preparation) and that builds on Wild (2011).

Denote by $M_{i}(t)$ the random number of patches in the population with $i \in I=\{1,2, \ldots, N\}$ mutants at demographic time $t$, and let $\mathbf{M}(t)=\left(M_{1}(t), \ldots, M_{N}(t)\right)$ be the associated random vector.

Starting with a single initial mutant in the focal patch at time $t=0$, i.e., $\mathbf{M}(0)=$ $(1,0, \ldots, 0)$, we are interested in finding an operational necessary and sufficient condition for the mutant type to go extinct in finite time with probability one; formally, a condition for $\operatorname{Pr}[\mathbf{M}(t)=\mathbf{0}$ for some $t \in \mathbb{N} \mid \mathbf{M}(0)=(1,0, \ldots, 0)]=1$. To that end, we first note that our assumption that there is an infinite number of islands implies that the stochastic process $\{\mathbf{M}(t)\}_{t \in \mathbb{N}}$ is a multi-type branching process (Wild, 2011), which is equivalent to assuming that only residents immigrate to the focal patch when the mutant is globally rare. Because we are only interested in characterizing extinction, it is sufficient to focus on matrix $\mathbf{A}(\tau, \theta)$ whose $(i, j)$ entry is the expected number of patches with $i \in I$ mutants (of type $\tau$ ) that are produced over one demographic time period by the focal patch when this has $j \in I$ mutants and when the population is otherwise monomorphic for type $\theta$. It then follows from standard results on multi-type branching processes (Karlin and Taylor, 1975, p. 412) that $\operatorname{Pr}[\mathbf{M}(t)=\mathbf{0}$ for some $t \mid \mathbf{M}(0)=(1,0, \ldots, 0)]=1$ if and only if the leading eigenvalue of $\mathbf{A}(\tau, \theta)$ is less than or equal to 1, i.e., if, and only if $\rho(\mathbf{A}(\tau, \theta)) \leq 1$ where $\rho(\mathbf{A}(\tau, \theta))$ denotes the spectral radius of $\mathbf{A}(\tau, \theta)$. It thus remains to (a) find an expression for $\mathbf{A}(\tau, \theta)$ under our biological assumptions, and (b) show that $\rho(\mathbf{A}(\tau, \theta)) \leq 1$ is equivalent to $W(\tau, \theta) \leq W(\theta, \theta)$. 
Following our life-cycle assumptions, one can write

$$
\mathbf{A}(\tau, \theta)=\mathbf{Q}(\tau, \theta)+\mathbf{E}(\tau, \theta)
$$

where $\mathbf{Q}(\tau, \theta)$ is the matrix for which the component in row $i$ and column $j$ is the probability that the focal patch with $j \in I$ mutants turns into a patch with $i \in I$ mutants, and where the transition probabilities are independent of the state $\mathbf{M}$. Thus, $\mathbf{Q}(\tau, \theta)$ is the transient matrix of the Markov chain, describing the subpopulation of mutants in the focal patch, with state space $\{0,1,2, \ldots, N\}$. This Markov chain has the local extinction of the mutant type as its unique absorbing state. We also have that,

$$
\mathbf{E}(\tau, \theta)=\left(\begin{array}{cccc}
\epsilon_{1}(\tau, \theta) & \epsilon_{2}(\tau, \theta) & \ldots & \epsilon_{N}(\tau, \theta) \\
0 & 0 & \ldots & 0 \\
\vdots & \vdots & \ddots & \vdots \\
0 & 0 & \ldots & 0
\end{array}\right),
$$

where $\epsilon_{j}(\tau, \theta)$ is the expected number of patches with one mutant that are produced by mutant emigration from the focal patch, when the focal patch is in state $j$. All other entries of matrix $\mathbf{E}(\tau, \theta)$ equal zero since when the number of islands is infinite, the probability that two or more offspring from the same patch settle on the same island through dispersal is zero. To see this, note what happens in the case where the number of patches is finite: then the probability that a given breeding spot on a given patch is settled through dispersal by an offspring of an individual from the focal patch is of order $O(m /(N D))$, where $D$ is the number of patches. The probability that two or more such offspring settle in the same patch is of order $O\left(m^{2} /(N D)^{2}\right)$ or smaller. Summing over all patches, the probability that two or more offspring from the same individual settle on the same patch through dispersal is thus at most of order $m^{2} /\left(N^{2} D\right)$, and hence goes to zero as $D \rightarrow \infty$. Therefore, the focal patch with $j$ mutants can only turn a patch with zero mutants into one with a single mutant.

Since

$$
\rho(\mathbf{A}(\tau, \theta)) \leq 1 \Longleftrightarrow \rho(\mathbf{A}(\tau, \theta)-\mathbf{I}) \leq 0
$$

Using eq. (A-1), we have

$$
\rho(\mathbf{A}(\tau, \theta)-\mathbf{I}) \leq 0 \Longleftrightarrow \rho(\mathbf{E}(\tau, \theta)-(\mathbf{I}-\mathbf{Q}(\tau, \theta))) \leq 0 .
$$


The matrix $\mathbf{I}-\mathbf{Q}(\tau, \theta)$ is non-negative, since all components of $\mathbf{Q}$ are between zero and one. In addition, $\mathbf{A}(\tau, \theta)$ - I has non-negative off-diagonal entries, and $\mathbf{E}(\tau, \theta)$ is non-negative. Therefore, we can apply the first-generation-theorem (Thieme, 2009, Theorem 2.1) to obtain the equivalence

$$
\rho(\mathbf{A}(\tau, \theta)-\mathbf{I}) \leq 0 \Longleftrightarrow \rho\left(\mathbf{E}(\tau, \theta)(\mathbf{I}-\mathbf{Q}(\tau, \theta))^{-1}\right) \leq 1
$$

By construction of $\mathbf{E}(\tau, \theta)$, the matrix $\mathbf{E}(\tau, \theta)(\mathbf{I}-\mathbf{Q}(\tau, \theta))^{-1}$ is upper triangular, and all its diagonal elements except the first are zero. Since the eigenvalues of a triangular matrix equal its diagonal entries, the leading eigenvalue of $\mathbf{E}(\tau, \theta)(\mathbf{I}-\mathbf{Q}(\tau, \theta))^{-1}$ equals its first diagonal element. This is given by $\sum_{k=1}^{N} \epsilon_{k}(\tau, \theta) t_{k}(\tau, \theta)$, where $t_{k}(\tau, \theta)$ is the expected number of time steps (sojourn time) that a patch that started with a single mutant spends with $k$ mutants (owing to the fact that the component $(i, j)$ of matrix $(\mathbf{I}-\mathbf{Q}(\tau, \theta))^{-1}$ corresponds to the expected sojourn time of the Markov chain in state $i$ when initially starting the process in state $j$ and excluding mutant immigration, Grinstead and Snell, 1997). Therefore,

$$
\rho(\mathbf{A}(\tau, \theta)) \leq 1 \Longleftrightarrow \sum_{k=1}^{N} \epsilon_{k}(\tau, \theta) t_{k}(\tau, \theta) \leq 1 .
$$

Condition (A-6) is equivalent to the non-invasibility condition for the mutant proposed by Metz and Gyllenberg (2001) for continuous-time processes and Ajar (2003) for discretetime processes, and proven to guarantee the local asymptotic instability of the deterministic dynamical system describing the growth of the mutant type when rare under a continuous time process (Massol et al., 2009).

We now proceed to re-write condition (A-6) in terms of fitness. The expected number of descendants of a single mutant $(\tau)$ individual in the focal patch in state $k \in I$ (that is, the personal fitness of a mutant in a patch with $k$ mutants), conditional on the rest of the population being monomorphic for $\theta$, can be written as

$$
w_{k}(\tau, \theta)=\phi_{k}(\tau, \theta)+\epsilon_{k}(\tau, \theta) / k,
$$

where $\phi_{k}(\tau, \theta)$ denotes the expected number of descendants in the focal patch produced through philopatry by a single mutant in the focal patch in state $k \in I$, conditional on the rest 
of the population being monomorphic for $\theta$, while $\epsilon_{k}(\tau, \theta) / k$ is the corresponding expected number of emigrant offspring produced by a single mutant. Then, because $\sum_{k=1}^{N} k t_{k}(\tau, \theta)$ counts the total local number of mutants during the lifespan of the lineage, and this is equal to $1+\sum_{k=1}^{N} \phi_{k}(\tau, \theta) k t_{k}(\tau, \theta)$ (the founding mutant plus the total number of local descendants, Mullon and Lehmann, 2014), we have, from eq. (A-7), the equality

$$
\sum_{k=1}^{N} \epsilon_{k}(\tau, \theta) t_{k}(\tau, \theta)-1=\sum_{k=1}^{N}\left[w_{k}(\tau, \theta)-1\right] k t_{k}(\tau, \theta) .
$$

Setting

$$
\Lambda(\tau, \theta)=\sum_{k=1}^{N}\left[w_{k}(\tau, \theta)-1\right] k t_{k}(\tau, \theta)
$$

we have

$$
\rho(\mathbf{A}(\tau, \theta)) \leq 1 \Longleftrightarrow \Lambda(\tau, \theta) \leq 0
$$

Since we assume no class structure (no roles) within patches, individuals of a given type are exchangeable within patches and types can be allocated randomly to neighbors of a focal individual. Because of the symmetry of the personal fitness function $w\left(\theta_{i}, \boldsymbol{\theta}_{-i}, 1_{\theta}\right)$, we can write

$$
w_{k}(\tau, \theta)=\sum_{\boldsymbol{\theta}_{-i} \in S_{k}(\tau, \theta)}\left(\begin{array}{c}
N-1 \\
k-1
\end{array}\right)^{-1} w\left(\tau, \boldsymbol{\theta}_{-i}, 1_{\theta}\right),
$$

where $S_{k}(\tau, \theta)$ is the set of all subsets of $\{\tau, \theta\}^{N-1}$ with exactly $k-1$ individuals having type $\tau$. Substituting into eq. A-9, and denoting by $\bar{t}(\tau, \theta)=\sum_{k=1}^{N} k t_{k}(\tau, \theta)$ the total sojourn time, produces

$$
\begin{aligned}
\Lambda(\tau, \theta) & =\sum_{k=1}^{N}\left[\sum_{\boldsymbol{\theta}_{-i} \in S_{k}(\tau, \theta)} \frac{w\left(\tau, \boldsymbol{\theta}_{-i}, 1_{\theta}\right)}{\left(\begin{array}{l}
N-1 \\
k-1
\end{array}\right)}-1\right] k t_{k}(\tau, \theta) \\
& =\sum_{k=1}^{N} \sum_{\boldsymbol{\theta}_{-i} \in S_{k}(\tau, \theta)}\left[w\left(\tau, \boldsymbol{\theta}_{-i}, 1_{\theta}\right) \frac{k t_{k}(\tau, \theta)}{\left(\begin{array}{l}
N-1 \\
k-1
\end{array}\right)}\right]-\sum_{k=1}^{N} k t_{k}(\tau, \theta) \\
& =\bar{t}(\tau, \theta)\left[\sum_{k=1}^{N} \sum_{\boldsymbol{\theta}_{-i} \in S_{k}(\tau, \theta)} w\left(\tau, \boldsymbol{\theta}_{-i}, 1_{\theta}\right) q_{k}(\tau, \theta)-1\right]
\end{aligned}
$$

where we used

$$
q_{k}(\tau, \theta)=\left(\begin{array}{c}
N-1 \\
k-1
\end{array}\right)^{-1} \frac{k t_{k}(\tau, \theta)}{\bar{t}(\tau, \theta)}
$$


Setting

$$
W(\tau, \theta)=\sum_{k=1}^{N} \sum_{\boldsymbol{\theta}_{-i} \in S_{k}(\tau, \theta)} q_{k}(\tau, \theta) w\left(\tau, \boldsymbol{\theta}_{-i}, 1_{\theta}\right)
$$

we finally obtain

$$
\Lambda(\tau, \theta)=\bar{t}(\tau, \theta)[W(\tau, \theta)-1] .
$$

Since $W(\theta, \theta)=1$, and since $\bar{t}(\tau, \theta) \geq 1$, it follows that

$$
\Lambda(\tau, \theta) \leq 0 \Longleftrightarrow W(\tau, \theta) \leq W(\theta, \theta)
$$

\section{Appendix B: univadability and maximizing behavior}

\section{First-order conditions}

We here prove that the inclusive fitness effect must be nil for a strategy $x$ to be uninvadable, or to be a symmetric Nash equilibrium strategy under any of the two goal functions, $u_{\mathrm{A}}$ or $u_{\mathrm{B}}$. As noted in the main text, eq. 4 implies that for $x$ to be uninvadable it must be that, given $x, y=x$ is a local maximum of

$$
W(y, x)=\sum_{k=1}^{N} \sum_{\boldsymbol{x}_{-i} \in S_{k}(y, x)} q_{k}(y, x) w\left(y, \boldsymbol{x}_{-i}, 1_{x}\right) .
$$

The first step of the proof consists in showing that the expression for $\partial W(y, x) /\left.\partial y\right|_{y=x}$ is the inclusive fitness effect (eq. 8 of the main text). We begin by noting that thanks to the permutation invariance of $w$ with respect to the components of $\boldsymbol{x}_{-i}$, for any $\boldsymbol{x}_{-i} \in S_{k}(y, x)$, we can write $\boldsymbol{x}_{-i}=\left(\mathbf{y}^{(k-1)}, \mathbf{x}^{(N-k)}\right)$, where $\mathbf{y}^{(k-1)}$ is the $(k-1)$-dimensional vector whose components all equal $y$, and $\mathbf{x}^{(N-k)}$ is the $(N-k)$-dimensional vector whose components all equal $x$. By a slight abuse of notation, we drop the parentheses around $\mathbf{y}^{(k-1)}, \mathbf{x}^{(N-k)}$, and write

$$
w\left(y, \boldsymbol{x}_{-i}, 1_{x}\right)=w\left(y, \mathbf{y}^{(k-1)}, \mathbf{x}^{(N-k)}, 1_{x}\right)
$$

Using this notation,

$$
W(y, x)=\sum_{k=1}^{N}\left(\begin{array}{c}
N-1 \\
k-1
\end{array}\right) q_{k}(y, x) w\left(y, \mathbf{y}^{(k-1)}, \mathbf{x}^{(N-k)}, 1_{x}\right) .
$$


Writing $w_{j}$ for the partial derivative of $w$ with respect to its $j$-th argument, where $j=$ $1, \ldots, N$, we have

$$
\begin{array}{r}
\frac{\partial W(y, x)}{\partial y}=\sum_{k=1}^{N}\left[\left(\begin{array}{c}
N-1 \\
k-1
\end{array}\right) \frac{\partial q_{k}(y, x)}{\partial y} w\left(y, \mathbf{y}^{(k-1)}, \mathbf{x}^{(N-k)}, 1_{x}\right)\right]+ \\
\sum_{k=1}^{N}\left[\left(\begin{array}{c}
N-1 \\
k-1
\end{array}\right) q_{k}(y, x) \sum_{j=1}^{k} w_{j}\left(y, \mathbf{y}^{(k-1)}, \mathbf{x}^{(N-k)}, 1_{x}\right)\right] .
\end{array}
$$

Noting that for $y=x, w\left(y, \mathbf{y}^{(k-1)}, \mathbf{x}^{(N-k)}, 1_{x}\right)=w\left(x, \mathbf{x}^{(N-1)}, 1_{x}\right)$, which is independent of $k$ so that it can be factored out in the first term, and using

$$
p_{k}(y, x)=\frac{k t_{k}(y, x)}{\bar{t}(y, x)}
$$

(see Box 2), we obtain

$$
\begin{aligned}
\left.\frac{\partial W(y, x)}{\partial y}\right|_{y=x}= & w\left(x, \mathbf{x}^{(N-1)}, 1_{x}\right) \sum_{k=1}^{N}\left[\left.\frac{\partial p_{k}(y, x)}{\partial y}\right|_{y=x}\right]+ \\
& \left.\sum_{k=1}^{N}\left[p_{k}(y, x) \sum_{j=1}^{k} w_{j}\left(y, \mathbf{y}^{(k-1)}, \mathbf{x}^{(N-k)}, 1_{x}\right)\right]\right|_{y=x} .
\end{aligned}
$$

This expression can be further simplified by noting that

$$
\sum_{k=1}^{N}\left(\left.\frac{\partial p_{k}(y, x)}{\partial y}\right|_{y=x}\right)=\left.\frac{\partial}{\partial y}\left(\sum_{k=1}^{N} p_{k}(y, x)\right)\right|_{y=x}=\left.\frac{\partial}{\partial y}(1)\right|_{y=x}=0 .
$$

Hence,

$$
\left.\frac{\partial W(y, x)}{\partial y}\right|_{y=x}=\left.\sum_{k=1}^{N}\left[p_{k}(y, x) \sum_{j=1}^{k} w_{j}\left(y, \mathbf{y}^{(k-1)}, \mathbf{x}^{(N-k)}, 1_{x}\right)\right]\right|_{y=x} .
$$

Permutation invariance further implies that for any $j \geq 2, w_{j}\left(x, \mathbf{x}^{(N-1)}, 1_{x}\right)=w_{N}\left(x, \mathbf{x}^{(N-1)}, 1_{x}\right)$ (it's as if the individual whose marginal type change is under consideration were systematically labeled to appear as the last component in the vector $\left.\mathbf{x}^{(N-1)}\right)$. Noticing also that 


$$
\begin{aligned}
&\left.\sum_{k=1}^{N}\left[p_{k}(y, x) w_{1}\left(y, \mathbf{y}^{(k-1)}, \mathbf{x}^{(N-k)}, 1_{x}\right)\right]\right|_{y=x}=w_{1}\left(x, \mathbf{x}^{(N-1)}, 1_{x}\right), \text { we can write: } \\
&\left.\frac{\partial W(y, x)}{\partial y}\right|_{y=x}=w_{1}\left(x, \mathbf{x}^{(N-1)}, 1_{x}\right)+\left.\sum_{k=2}^{N}\left[p_{k}(y, x) \sum_{j=2}^{k} w_{j}\left(y, \mathbf{y}^{(k-1)}, \mathbf{x}^{(N-k)}, 1_{x}\right)\right]\right|_{y=x} \\
&=w_{1}\left(x, \mathbf{x}^{(N-1)}, 1_{x}\right)+\sum_{k=2}^{N}\left[p_{k}(x, x)(k-1) w_{N}\left(x, \mathbf{x}^{(N-1)}, 1_{x}\right)\right] \\
&=w_{1}\left(x, \mathbf{x}^{(N-1)}, 1_{x}\right)+ \\
&(N-1) w_{N}\left(x, \mathbf{x}^{(N-1)}, 1_{x}\right) \sum_{k=2}^{N}\left[\frac{(k-1) p_{k}(x, x)}{(N-1)}\right] \\
&=w_{1}\left(x, \mathbf{x}^{(N-1)}, 1_{x}\right)+r(x, x)(N-1) w_{N}\left(x, \mathbf{x}^{(N-1)}, 1_{x}\right)
\end{aligned}
$$

where in the last line we used the definition of relatedness given in Box 2. This last line is the inclusive fitness effect (eq. 8 of the main text), and where in the main text we used the notation $\boldsymbol{x}_{x}$ for $\mathbf{x}^{(N-1)}$.

Turning now to the goal functions, we start with the $u_{\mathrm{A}}$ function defined in eq. 6 of the main text. A necessary condition for a strategy $x$ to be a symmetric Nash equilibrium strategy of $G_{\mathrm{A}}=\left(\mathbb{N}, X, u_{\mathrm{A}}\right)$ is that, if all the other players except player $i$ use strategy $x$, strategy $x$ satisfy the first-order condition for a local maximum for individual $i$ :

$$
\left.\frac{\partial u_{\mathrm{A}}\left(x_{i}, \mathbf{x}^{(N-1)}, \phi\right)}{\partial x_{i}}\right|_{x_{i}=x}=0
$$

Note that in the second term in the $u_{A}$ goal function (eq. 6 of the main text), $x_{i}$ appears exactly once in $\boldsymbol{x}_{-j}$, for each $j$. By permutation invariance, we can without loss of generality assume that $x_{i}$ appears as the last component in each $\boldsymbol{x}_{-j}$, so that, for each $j$, the partial derivative of $w\left(x_{j}, \boldsymbol{x}_{-j}, \phi\right)$ with respect to $x_{i}$ writes $w_{N}\left(x_{j}, \boldsymbol{x}_{-j}, \phi\right)$. Moreover, since $\bar{x}=x$ if all other individuals uses strategy $x$, we obtain

$$
\left.\frac{\partial u_{\mathrm{A}}\left(x_{i}, \mathbf{x}^{(N-1)}, \phi\right)}{\partial x_{i}}\right|_{x_{i}=x}=w_{1}\left(x, \mathbf{x}^{(N-1)}, 1_{x}\right)+r(x, x)(N-1) w_{N}\left(x, \mathbf{x}^{(N-1)}, 1_{x}\right),
$$

which coincides with the inclusive fitness effect (eq. 8 of the main text).

Next, we turn to the $u_{\mathrm{B}}$ goal function defined in eq. 7 of the main text. A necessary condition for a strategy $x$ to be a symmetric Nash equilibrium strategy of $G_{\mathrm{B}}=\left(\mathbb{N}, X, u_{\mathrm{B}}\right)$ 
is that, if all the other players except player $i$ use strategy $x$, i.e., if $\boldsymbol{x}_{-i}=\mathbf{x}^{(N-1)}$, strategy $x$ satisfy the first-order condition for a local maximum for individual $i$ :

$$
\left.\frac{\partial u_{\mathrm{B}}\left(x_{i}, \mathbf{x}^{(N-1)}, \phi\right)}{\partial x_{i}}\right|_{x_{i}=x}=0 .
$$

Permutation invariance implies

$$
u_{\mathrm{B}}\left(x_{i}, \mathbf{x}^{(N-1)}, \phi\right)=\sum_{k=1}^{N} q_{k}(\bar{x}, \bar{x})\left(\begin{array}{c}
N-1 \\
k-1
\end{array}\right) w\left(x_{i}, \mathbf{x}_{i}^{(k-1)}, \mathbf{x}^{(N-k)}, \phi\right) .
$$

Supposing now that everyone in the population except individual $i$ uses strategy $x$, and applying observations made earlier in this proof, we obtain

$$
\begin{aligned}
\left.\frac{\partial u_{\mathrm{B}}\left(x_{i}, \mathbf{x}^{(N-1)}, \phi\right)}{\partial x_{i}}\right|_{x_{i}=x} & =\left.\left[\sum_{k=1}^{N} q_{k}(x, x)\left(\begin{array}{c}
N-1 \\
k-1
\end{array}\right) \sum_{j=1}^{k} w_{j}\left(x_{i}, \mathbf{x}_{i}^{(k-1)}, \mathbf{x}^{(N-k)}, \phi\right)\right]\right|_{x_{i}=x} \\
& =w_{1}\left(x, \mathbf{x}^{(N-1)}, 1_{x}\right)+(N-1) w_{N}\left(x, \mathbf{x}^{(N-1)}, 1_{x}\right) \sum_{k=2}^{N} \frac{(k-1) p_{k}(x, x)}{(N-1)} \\
& =w_{1}\left(x, \mathbf{x}^{(N-1)}, 1_{x}\right)+r(x, x)(N-1) w_{N}\left(x, \mathbf{x}^{(N-1)}, 1_{x}\right), \quad(\mathrm{B}-14)
\end{aligned}
$$

which again coincides with the inclusive fitness effect (eq. 8 of the main text).

\section{Second-order conditions}

We here prove the two results in Box 3 by evaluating the second-order conditions for uninvadability and for the symmetric Nash equilibrium under $u_{\mathrm{A}}$ and $u_{\mathrm{B}}$.

\section{Lineage fitness}

Suppose that $X_{\mathrm{D}}=\{x\}$, for some $x \in X$. We have

$$
\begin{aligned}
\frac{\partial^{2} W(y, x)}{\partial y^{2}} & =\sum_{k=1}^{N}\left[\left(\begin{array}{c}
N-1 \\
k-1
\end{array}\right) \frac{\partial^{2} q_{k}(y, x)}{\partial y^{2}} w\left(y, \mathbf{y}^{(k-1)}, \mathbf{x}^{(N-k)}, 1_{x}\right)\right]+ \\
& 2 \sum_{k=1}^{N}\left[\left(\begin{array}{c}
N-1 \\
k-1
\end{array}\right) \frac{\partial q_{k}(y, x)}{\partial y} \sum_{j=1}^{k} w_{j}\left(y, \mathbf{y}^{(k-1)}, \mathbf{x}^{(N-k)}, 1_{x}\right)\right]+ \\
& \sum_{k=1}^{N}\left[\left(\begin{array}{c}
N-1 \\
k-1
\end{array}\right) q_{k}(y, x) \sum_{j=1}^{k} \sum_{\ell=1}^{k} w_{j \ell}\left(y, \mathbf{y}^{(k-1)}, \mathbf{x}^{(N-k)}, 1_{x}\right)\right]
\end{aligned}
$$


As noted above, we need to evaluate this expression at $y=x$. Since $\left.w\left(y, \mathbf{y}^{(k-1)}, \mathbf{x}^{(N-k)}, 1_{x}\right)\right|_{y=x}=$ $w\left(x, \mathbf{x}^{(N-1)}, 1_{x}\right)$, which is independent of $k$, and given the definition of $q_{k}(y, x)$, when evaluated at $y=x$, the first line in eq. B-15 may be written as

$$
\begin{aligned}
& \left.w\left(x, \mathbf{x}^{(N-1)}, 1_{x}\right) \sum_{k=1}^{N} \frac{\partial^{2} p_{k}(y, x)}{\partial y^{2}}\right|_{y=x} \\
& =\left.w\left(x, \mathbf{x}^{(N-1)}, 1_{x}\right) \frac{\partial^{2}}{\partial y^{2}} \sum_{k=1}^{N} p_{k}(y, x)\right|_{y=x} \\
& =w\left(x, \mathbf{x}^{(N-1)}, 1_{x}\right) \frac{\partial^{2}}{\partial y^{2}}(1)=0
\end{aligned}
$$

Next, and disregarding the constant 2, the second line in eq. B-15 may be rewritten as follows:

$$
\begin{aligned}
& \left.\sum_{k=1}^{N}\left[\left(\begin{array}{c}
N-1 \\
k-1
\end{array}\right) \frac{\partial q_{k}(y, x)}{\partial y} w_{1}\left(y, \mathbf{y}^{(k-1)}, \mathbf{x}^{(N-k)}, 1_{x}\right)\right]\right|_{y=x}+ \\
& \left.\sum_{k=2}^{N}\left[\left(\begin{array}{c}
N-1 \\
k-1
\end{array}\right) \frac{\partial q_{k}(y, x)}{\partial y} \sum_{j=2}^{k} w_{j}\left(y, \mathbf{y}^{(k-1)}, \mathbf{x}^{(N-k)}, 1_{x}\right)\right]\right|_{y=x} \\
& =\left.w_{1}\left(x, \mathbf{x}^{(N-1)}, 1_{x}\right) \frac{\partial}{\partial y} \sum_{k=1}^{N} p_{k}(y, x)\right|_{y=x}+ \\
& +\left.\sum_{k=2}^{N}\left[\left(\begin{array}{c}
N-1 \\
k-1
\end{array}\right) \frac{\partial q_{k}(y, x)}{\partial y}(k-1) w_{N}\left(x, \mathbf{x}^{(N-1)}, 1_{x}\right)\right]\right|_{y=x}
\end{aligned}
$$

The first term on the right-hand side of this equality equals zero (see eq. B-7). Turning now to the second term, by factoring out $w_{N}\left(x, \mathbf{x}^{(N-1)}, 1_{x}\right)$, by multiplying and dividing by $(N-1)$, and by using the definition of $q_{k}$ (see Box 2$)$, this term writes

$$
\begin{aligned}
& \left.(N-1) w_{N}\left(x, \mathbf{x}^{(N-1)}, 1_{x}\right) \sum_{k=2}^{N}\left[\frac{\partial p_{k}(y, x)}{\partial y} \frac{(k-1)}{(N-1)}\right]\right|_{y=x} \\
& =\left.(N-1) w_{N}\left(x, \mathbf{x}^{(N-1)}, 1_{x}\right) \frac{\partial}{\partial y} \sum_{k=2}^{N}\left[\frac{(k-1) p_{k}(y, x)}{(N-1)}\right]\right|_{y=x} \\
& =\left.(N-1) w_{N}\left(x, \mathbf{x}^{(N-1)}, 1_{x}\right) r_{1}(y, x)\right|_{y=x} .
\end{aligned}
$$

Finally, we proceed to rewriting the third line in the original expression (eq. B-15). Using 
permutation invariance, we obtain the following expression:

$$
\begin{aligned}
& w_{11}\left(x, \mathbf{x}^{(N-1)}, 1_{x}\right)+ \\
& \left.(N-1) w_{1 N}\left(x, \mathbf{x}^{(N-1)}, 1_{x}\right) \sum_{k=1}^{N}\left[\frac{(k-1) p_{k}(y, x)}{(N-1)}\right]\right|_{y=x}+ \\
& \left.(N-1) w_{N 1}\left(x, \mathbf{x}^{(N-1)}, 1_{x}\right) \sum_{k=1}^{N}\left[\frac{(k-1) p_{k}(y, x)}{(N-1)}\right]\right|_{y=x}+ \\
& \left.(N-1) w_{N N}\left(x, \mathbf{x}^{(N-1)}, 1_{x}\right) \sum_{k=1}^{N}\left[\frac{(k-1) p_{k}(y, x)}{(N-1)}\right]\right|_{y=x}+ \\
& \left.(N-2)(N-1) w_{2 N}\left(x, \mathbf{x}^{(N-1)}, 1_{x}\right) \sum_{k=1}^{N}\left[\frac{(k-2)(k-1) p_{k}(y, x)}{(N-2)(N-1)}\right]\right|_{y=x} .
\end{aligned}
$$

Using the coefficient of pairwise relatedness, $r(y, x)$, as well as the coefficient of triplet relatedness,

$$
\tilde{r}(y, x)=\sum_{k=1}^{N} \frac{(k-2)(k-1)}{(N-2)(N-1)} p_{k}(y, x),
$$

and recalling that $w_{1 N}=w_{N 1}$, the third line expression in eq. B-15 may thus be written:

$$
\begin{aligned}
& w_{11}\left(x, \mathbf{x}^{(N-1)}, 1_{x}\right)+2(N-1) w_{1 N}\left(x, \mathbf{x}^{(N-1)}, 1_{x}\right) r(x, x)+ \\
& (N-1) w_{N N}\left(x, \mathbf{x}^{(N-1)}, 1_{x}\right) r(x, x)+ \\
& (N-2)(N-1) w_{2 N}\left(x, \mathbf{x}^{(N-1)}, 1_{x}\right) \tilde{r}(x, x) .
\end{aligned}
$$

Collecting the expressions for the second and third lines in eq. B-15 (respectively in eq. B18 and eq. B-21), and writing $r_{1}(x, x)$ for $\left.r_{1}(y, x)\right|_{y=x}$, the expression in eq. B-15 writes:

$$
\begin{aligned}
\left.\frac{\partial^{2} W(y, x)}{\partial y^{2}}\right|_{y=x} & =w_{11}\left(x, \mathbf{x}^{(N-1)}, 1_{x}\right)+r(x, x)(N-1) w_{N N}\left(x, \mathbf{x}^{(N-1)}, 1_{x}\right)+ \\
& r(x, x) 2(N-1) w_{1 N}\left(x, \mathbf{x}^{(N-1)}, 1_{x}\right)+ \\
& \tilde{r}(x, x)(N-2)(N-1) w_{2 N}\left(x, \mathbf{x}^{(N-1)}, 1_{x}\right)+ \\
& r_{1}(x, x) 2(N-1) w_{N}\left(x, \mathbf{x}^{(N-1)}, 1_{x}\right)
\end{aligned}
$$

which is consistent with eq. 9 of Ajar (2003) and eq. 29 of Wakano and Lehmann (2014). 


\section{Goal function $u_{\mathrm{A}}$}

We now turn to the $u_{\mathrm{A}}$ goal function. By Result 1, and given that $X_{\mathrm{D}}$ is a singleton, $X_{\mathrm{D}}=\{x\}$, a necessary condition for $x$ to be a symmetric Nash equilibrium strategy in the population game $G_{\mathrm{A}}=\left(\mathbb{N}, X, u_{\mathrm{A}}\right)$ is $\partial^{2} u_{\mathrm{A}}\left(x_{i}, \boldsymbol{x}_{-i}, \phi\right) /\left.\partial x_{i}^{2}\right|_{x_{i}=x} \leq 0$, and a sufficient condition is that this inequality hold strictly. By permutation invariance, we can without loss of generality assume that $x_{i}$ appears as the last component in each $\boldsymbol{x}_{-j}$ in the expression for $u_{\mathrm{A}}$ (see definition of $u_{\mathrm{A}}$ in the main text) for each $j$, the partial derivative of $w\left(x_{j}, \boldsymbol{x}_{-j}, \phi\right)$ with respect to $x_{i}$ writes $w_{N}\left(x_{j}, \boldsymbol{x}_{-j}, \phi\right)$, and the second-order partial derivative with respect to $x_{i}$ writes $w_{N N}\left(x_{j}, \boldsymbol{x}_{-j}, \phi\right)$. Moreover, since $\bar{x}=x$ if all other individuals use strategy $x$ in eq. 6 of the main text, we immediately obtain

$$
\left.\frac{\partial^{2} u_{\mathrm{A}}\left(x_{i}, \boldsymbol{x}_{-i}, \phi\right)}{\partial x_{i}^{2}}\right|_{x_{i}=x}=w_{11}\left(x, \mathbf{x}^{(N-1)}, 1_{x}\right)+r(x, x)(N-1) w_{N N}\left(x, \mathbf{x}^{(N-1)}, 1_{x}\right) .
$$

Suppose now that $x \in X_{\mathrm{U}}$; then $\partial^{2} W(y, x) /\left.\partial y^{2}\right|_{y=x} \leq 0$. By comparing eq. B-22 and eq. B-23, it immediately follows that if the sum of the three last terms in eq. B-22 is strictly positive, $\partial^{2} u_{\mathrm{A}}\left(x_{i}, \boldsymbol{x}_{-i}, \phi\right) /\left.\partial x_{i}^{2}\right|_{x_{i}=x}<0$, in which case $x \in X_{\mathrm{E}}\left(u_{\mathrm{A}}\right)$. The conditions stated in part (a) of Result 3 in Box 3 are sufficient for the sum of the three last terms in eq. B-22 to be strictly positive.

Suppose now that $x \in X_{\mathrm{E}}\left(u_{\mathrm{A}}\right)$; then $\partial^{2} u_{\mathrm{A}}\left(x_{i}, \boldsymbol{x}_{-i}, \phi\right) /\left.\partial x_{i}^{2}\right|_{x_{i}=x} \leq 0$. By comparing eq. B22 and eq. B-23, it immediately follows that if the sum of the three last terms in eq. B-22 is strictly negative, $\partial^{2} W(y, x) /\left.\partial y^{2}\right|_{y=x}<0$, in which case $x \in X_{\mathrm{U}}$. The conditions stated in part (b) of Result 3 in Box 3 are sufficient for the sum of the three last terms in eq. B-22 to be strictly negative.

Finally, if the sum of the three last terms in eq. B-22 equals zero,

$$
\left.\frac{\partial^{2} W(y, x)}{\partial y^{2}}\right|_{y=x}=\left.\frac{\partial^{2} u_{\mathrm{A}}\left(x_{i}, \boldsymbol{x}_{-i}, \phi\right)}{\partial x_{i}^{2}}\right|_{x_{i}=x}
$$

in which case $X_{\mathrm{E}}\left(u_{\mathrm{A}}\right)=X_{\mathrm{U}}$. The conditions stated in part (c) of Result 3 in Box 3 are sufficient for the sum of the three last terms in eq. B-22 to equal zero. 


\section{Goal function $u_{\mathrm{B}}$}

The proof is qualitatively similar to the previous one. By Result 1 , and given that $X_{\mathrm{D}}$ is a singleton, $X_{\mathrm{D}}=\{x\}$, a necessary condition for $x$ to be a symmetric Nash equilibrium strategy in the population game $G_{\mathrm{B}}=\left(\mathbb{N}, X, u_{\mathrm{B}}\right)$ is $\partial^{2} u_{\mathrm{B}}\left(x_{i}, \boldsymbol{x}_{-i}, \phi\right) /\left.\partial x_{i}^{2}\right|_{x_{i}=x} \leq 0$, and a sufficient condition is that this inequality hold strictly. By permutation invariance, we can without loss of generality assume that $x_{i}$ appears as the last component in each $\boldsymbol{x}_{-j}$ in the expression for $u_{\mathrm{B}}$ (see eq. 7 of the main text), so that, for each $j$, the partial derivative of $w\left(x_{j}, \boldsymbol{x}_{-j}, \phi\right)$ with respect to $x_{i}$ writes $w_{N}\left(x_{j}, \boldsymbol{x}_{-j}, \phi\right)$, and the second-order partial derivative with respect to $x_{i}$ writes $w_{N N}\left(x_{j}, \boldsymbol{x}_{-j}, \phi\right)$. Moreover, since $\bar{x}=x$ if all other individuals use strategy $x$, we immediately obtain

$$
\begin{aligned}
\frac{\partial^{2} u_{\mathrm{B}}\left(x_{i}, \boldsymbol{x}_{-i}, \phi\right)}{\partial x_{i}^{2}} & =w_{11}\left(x, \mathbf{x}^{(N-1)}, 1_{x}\right)+r(x, x)(N-1) w_{N N}\left(x, \mathbf{x}^{(N-1)}, 1_{x}\right)+ \\
& r(x, x) 2(N-1) w_{1 N}\left(x, \mathbf{x}^{(N-1)}, 1_{x}\right)+ \\
& \tilde{r}(x, x)(N-2)(N-1) w_{2 N}\left(x, \mathbf{x}^{(N-1)}, 1_{x}\right)
\end{aligned}
$$

Suppose now that $x \in X_{\mathrm{U}}$; then $\partial^{2} W(y, x) /\left.\partial y^{2}\right|_{y=x} \leq 0$. By comparing eq. B-22 and eq. B-25, it immediately follows that if the last term in eq. B-22 is strictly positive, i.e., if the condition stated in part (a) of Result 4 in Box 3 is satisfied, $\partial^{2} u_{\mathrm{B}}\left(x_{i}, \boldsymbol{x}_{-i}, \phi\right) /\left.\partial x_{i}^{2}\right|_{x_{i}=x}<0$, in which case $x \in X_{\mathrm{E}}\left(u_{\mathrm{B}}\right)$.

Suppose now that $x \in X_{\mathrm{E}}\left(u_{\mathrm{B}}\right)$; then $\partial^{2} u_{\mathrm{B}}\left(x_{i}, \boldsymbol{x}_{-i}, \phi\right) /\left.\partial x_{i}^{2}\right|_{x_{i}=x} \leq 0$. By comparing eq. B22 and eq. B-25, it immediately follows that if the last term in eq. B-22 is strictly negative, i.e., if the condition stated in part (b) of Result 4 in Box 3 is satisfied, $\partial^{2} W(y, x) /\left.\partial y^{2}\right|_{y=x}<0$, in which case $x \in X_{\mathrm{U}}$.

Finally, if the last term in eq. B-22 equals zero, i.e., if the condition stated in part (c) of Result 4 in Box 3 is satisfied, $\partial^{2} W(y, x) /\left.\partial y^{2}\right|_{y=x}=\partial^{2} u_{\mathrm{B}}\left(x_{i}, \boldsymbol{x}_{-i}, \phi\right) /\left.\partial x_{i}^{2}\right|_{x_{i}=x}$, in which case $X_{\mathrm{E}}\left(u_{\mathrm{B}}\right)=X_{\mathrm{U}}$. 


\section{Appendix C: weak selection}

We here prove the weak selection results for uninvadability (eq. 14 of the main text) and maximizing behavior (eq. 16 of the main text). We start by evaluating personal fitness under weak selection. By using a first-order Taylor expansion of the fitness of a focal individual $i$ in a focal patch, with respect to $\delta$ and evaluated at $\delta=0$, we can write

$$
\begin{aligned}
w\left(\theta_{i}, \boldsymbol{\theta}_{-i}, 1_{\theta}\right)=1+\delta\left[a_{\mathrm{f}}\left(\pi\left(\theta_{i}, \boldsymbol{\theta}_{-i}, 1_{\theta}\right)-\pi\left(\boldsymbol{\theta}^{(N)}, 1_{\theta}\right)\right)\right. \\
\left.\quad-a_{\mathrm{n}} \sum_{j \neq i}\left(\frac{\pi\left(\theta_{j}, \boldsymbol{\theta}_{-j}, 1_{\theta}\right)-\pi\left(\boldsymbol{\theta}^{(N)}, 1_{\theta}\right)}{N-1}\right)\right]+O\left(\delta^{2}\right),
\end{aligned}
$$

where $a_{\mathrm{f}}$ and $a_{\mathrm{n}}$ are coefficients that depend on structural demographic parameters, such as patch size and migration rate, and $\boldsymbol{\theta}^{(N)}$ is the $N$-dimensional vector whose components all equal $\theta$. This expansion for fitness follows from four facts about $w\left(\theta_{i}, \boldsymbol{\theta}_{-i}, 1_{\theta}\right)$ : (i) to the first

order in $\delta$, fitness is necessarily an affine (linear plus constant) function in the payoff of each individual in the population; (ii) each individual $j \in I$ with $j \neq i$ has the same effect on the fitness of the focal individual $i$ (permutation invariance of payoff effects of neighbors); (iii) each individual from each patch different from the focal patch has the same effect on the fitness of focal $i$ (permutation invariance of payoff effects of individuals in different patches when they all carry $x$ ); and (iv) total selective effects (here total effects of payoff on fitness) must sum to zero in a monomorphic population, as the expected change in type number or frequency is necessarily nil (Lehmann and Rousset, 2009, p. 38).

Owing to the assumption (introduced in section 2.3) that the fitness of an individual is monotonic increasing in its payoff and bounded by it, we have $0<a_{\mathrm{f}} \leq 1$. Owing to the assumption that the fitness of an individual is monotonic decreasing in the payoff of its patch neighbors, and that the negative effect on fitness of a single patch neighbor having its payoff varied is not larger than the positive effect of the focal having its own payoff varied, we have $0 \leq a_{\mathrm{n}} \leq a_{\mathrm{f}}$. Letting

$$
\lambda=a_{\mathrm{n}} / a_{\mathrm{f}}
$$


we conclude that $0 \leq \lambda \leq 1$. Factoring out $a_{\mathrm{f}}>0$ from eq. C-1, we obtain:

$$
w\left(\theta_{i}, \boldsymbol{\theta}_{-i}, 1_{\theta}\right)=1+\delta a_{\mathrm{f}}\left[\pi\left(\theta_{i}, \boldsymbol{\theta}_{-i}, 1_{\theta}\right)-\lambda \sum_{j \neq i} \frac{\pi\left(\theta_{j}, \boldsymbol{\theta}_{-j}, 1_{\theta}\right)}{N-1}-(1-\lambda) \pi\left(\boldsymbol{\theta}^{(N)}, 1_{\theta}\right)\right]+O\left(\delta^{2}\right) .
$$

This shows that the coefficient $\lambda$ quantifies the proportion of density-dependent competition that is local, among patch members, and thus defines the spatial scale of density-dependent competition (Frank, 1998, p. 115).

As an illustration, in the Moran island model, and thus using the fitness function given in Box 1 along with the fecundity function (eq. 9 of the main text, our corresponding deathfactor in Box 1), a Taylor expansion and subsequent rearrangement yields

$$
\lambda=\left\{\begin{array}{cc}
\frac{(N-1)(1-m)^{2}}{N-(1-m)^{2}} & \text { for fecundity effects } \\
1 & \text { for survival effects. }
\end{array}\right.
$$

We now note that when types only affect material payoff, vital rates (fecundity and survival) are the same for all types when $\delta=0$. Hence, also fitness is then type independent and thus equal to 1 (set $\delta=0$ in the fitness function in Box 1 when fecundity is given by eq. 9). All these quantities are then exchangeable variables between individuals; the population is monomorphic and the resulting evolutionary process is neutral (Crow and Kimura, 1970; Gillespie, 2004; Ewens, 2004). Under this neutral process, that is independent of resident type $\theta$, the experienced lineage-size distribution (see Box 2) takes a value determined solely by local sampling drift (see e.g., in Crow and Kimura, 1970; Ewens, 2004; Rousset, 2004 for an explicit example). We denote by $q_{k}^{\circ}$ the associated type-profile distribution, where the superscript o signifies that a quantity is evaluated at the neutral process when $\delta=0$. Hence, we can write

$$
q_{k}\left(\theta^{\prime}, \theta\right)=q_{k}^{\circ}+O(\delta)
$$

where $O(\delta)$ is the deviation (relative to the neutral process) of the type profile distribution induced by selection (i.e., $\delta>0$ ) that is at most of order $\delta$.

From eq. C-3 and eq. C-5 we have

$$
w\left(\theta^{\prime}, \boldsymbol{\theta}_{-i}, 1_{\theta}\right) q_{k}\left(\theta^{\prime}, \theta\right)=q_{k}\left(\theta^{\prime}, \theta\right)+\delta a_{\mathrm{f}}\left[\pi_{\mathrm{R}}\left(\theta^{\prime}, \boldsymbol{\theta}_{-i}, 1_{\theta}\right)-(1-\lambda) \pi\left(\boldsymbol{\theta}^{(N)}, 1_{\theta}\right)\right] q_{k}^{\circ}+O\left(\delta^{2}\right),
$$


where the notation of the payoff advantage

$$
\pi_{\mathrm{R}}\left(\theta_{i}, \boldsymbol{\theta}_{-i}, 1_{\theta}\right)=\pi\left(\theta_{i}, \boldsymbol{\theta}_{-i}, 1_{\theta}\right)-\frac{\lambda}{N-1} \sum_{j \neq i} \pi\left(\theta_{j}, \boldsymbol{\theta}_{-j}, 1_{\theta}\right),
$$

was introduced in the main text (eq. 13).

Substituting this into lineage fitness (eq. A-14) produces

$$
\begin{aligned}
W\left(\theta^{\prime}, \theta\right)= & \sum_{k=1}^{N} \sum_{\boldsymbol{\theta}_{-i} \in S_{k}\left(\theta^{\prime}, \theta\right)} w\left(\theta_{i}, \boldsymbol{\theta}_{-i}, 1_{\theta}\right) q_{k}\left(\theta^{\prime}, \theta\right) \\
& =1+\delta a_{\mathrm{f}} \sum_{k=1}^{N} \sum_{\boldsymbol{\theta}_{-i} \in S_{k}\left(\theta^{\prime}, \theta\right)}\left[\pi_{\mathrm{R}}\left(\theta^{\prime}, \boldsymbol{\theta}_{-i}, 1_{\theta}\right)-(1-\lambda) \pi\left(\boldsymbol{\theta}^{(N)}, 1_{\theta}\right)\right] q_{k}^{\circ}+O\left(\delta^{2}\right) .
\end{aligned}
$$

Hence, to the first order in selection intensity $\delta$, the expectation of fitness is taken over the neutral experienced lineage-size distribution, which is a common result of evolutionary dynamics that applies both to finite and infinite populations (Roze and Rousset, 2003; Rousset, 2004; Lehmann and Rousset, 2009; Lessard, 2009)

Combining eq. C-8 with the definition of lineage payoff (eq. 12 of the main text):

$$
\Pi\left(\theta^{\prime}, \theta\right)=\sum_{k=1}^{N} \sum_{\boldsymbol{\theta}_{-i} \in S_{k}\left(\theta^{\prime}, \theta\right)} \pi_{\mathrm{R}}\left(\theta^{\prime}, \boldsymbol{\theta}_{-i}, 1_{\theta}\right) q_{k}^{\circ},
$$

we can write lineage fitness as

$$
W\left(\theta^{\prime}, \theta\right)=1+\delta a_{\mathrm{f}}\left[\Pi\left(\theta^{\prime}, \theta\right)-(1-\lambda) \pi\left(\boldsymbol{\theta}^{(N)}, 1_{\theta}\right)\right]+O\left(\delta^{2}\right)
$$

Neglecting higher order terms in $\delta$ in this equation allows us to write the condition for uninvadability $[W(\tau, \theta) \leq W(\theta, \theta)$ for all $\tau \in \Theta]$ for weak selection as $\Pi(\tau, \theta) \leq \Pi(\theta, \theta)$ for all $\tau \in \Theta$, which implies that $\theta$ is uninvadable if and only if

$$
\theta \in \arg \max _{\tau \in \Theta} \Pi(\tau, \theta)
$$

From eq. C-11 a necessary and sufficient condition for $x$ to be uninvadable under weak selection is

$$
x \in \arg \max _{y \in X} \Pi(y, x)
$$


where

$$
\Pi(y, x)=\sum_{k=1}^{N} \sum_{\boldsymbol{x}_{-i} \in S_{k}(y, x)} q_{k}^{\circ} \pi_{\mathrm{R}}\left(y, \boldsymbol{x}_{-i}, 1_{x}\right),
$$

which, using the same notation as before, can be written as

$$
\begin{aligned}
\Pi(y, x) & =\sum_{k=1}^{N}\left(\begin{array}{c}
N-1 \\
k-1
\end{array}\right) q_{k}^{\circ} \pi_{\mathrm{R}}\left(y, \mathbf{y}^{(k-1)}, \mathbf{x}^{(N-k)}, 1_{x}\right) \\
& =\sum_{k=1}^{N} p_{k}^{\circ} \pi_{\mathrm{R}}\left(y, \mathbf{y}^{(k-1)}, \mathbf{x}^{(N-k)}, 1_{x}\right) .
\end{aligned}
$$

Hence, eq. C-12 writes

$$
x \in \arg \max _{y \in X} \sum_{k=1}^{N} p_{k}^{\circ} \pi_{\mathrm{R}}\left(y, \mathbf{y}^{(k-1)}, \mathbf{x}^{(N-k)}, 1_{x}\right) .
$$

We turn now to the goal function $u_{\mathrm{C}}$ defined in the main text (eq. 15), which is

$$
u_{\mathrm{C}}\left(x_{i}, \boldsymbol{x}_{-i}, \phi\right)=\sum_{k=1}^{N} \sum_{\tilde{\boldsymbol{x}}_{-i} \in P_{k}\left(\boldsymbol{x}_{-i}\right)} q_{k}^{\circ} \pi_{\mathrm{R}}\left(x_{i}, \tilde{\boldsymbol{x}}_{-i}, 1_{x}\right) .
$$

A strategy $x$ is a symmetric Nash equilibrium strategy of $G_{\mathrm{C}}=\left(\mathbb{N}, X, u_{\mathrm{C}}\right)$ if and only if it is optimal for each individual $i$ to play $x$ if all the other players also play $x$. Thus, and noting that if all the other players except player $i$ use strategy $x$ we can write $\boldsymbol{x}_{-i}=\mathbf{x}^{(N-1)}$, the necessary and sufficient condition for $x$ to be a symmetric Nash equilibrium strategy of $G_{\mathrm{C}}=\left(\mathbb{N}, X, u_{\mathrm{C}}\right)$ writes:

$$
x \in \arg \max _{x_{i} \in X} u_{\mathrm{C}}\left(x_{i}, \mathbf{x}^{(N-1)}, 1_{x}\right) .
$$

By permutation invariance,

$$
u_{\mathrm{C}}\left(x_{i}, \mathbf{x}^{(N-1)}, 1_{x}\right)=\sum_{k=1}^{N} p_{k}^{\circ} \pi_{\mathrm{R}}\left(x_{i}, \mathbf{x}_{i}^{(k-1)}, \mathbf{x}^{(N-k)}, 1_{x}\right)
$$

where $\mathbf{x}_{i}^{(k-1)}$ is the $(k-1)$-dimensional vector whose components all equal $x_{i}$. So eq. C-17 is identical with eq. C-15, which establishes Result 6. 


\section{Appendix D: Moran process calculations}

\section{Sojourn times}

We here evaluate the different results for our examples based on the Moran process (along similar lines as in Mullon and Lehmann, 2014). The key is to obtain an expression for $t_{i}(\tau, \theta)$, which is obtained from the (transient) transition matrix $\mathbf{Q}(\tau, \theta)$ (see eq. A-1) with element $q_{i j}(\tau, \theta)$ giving the probability that the focal patch with $j \in I=\{1,2, \ldots, N\}$ mutants turns into a patch. Since for a Moran process only one individual in a patch can be replaced per unit of demographic time, the Markov chain describing local lineage is a birth-death process (e.g, Karlin and Taylor, 1975), whose transition probabilities for transient states are

$$
q_{i j}(\tau, \theta)= \begin{cases}\tilde{b}_{j}(\tau, \theta), & \text { if } i=j+1 \text { ("birth" of a mutant) } \\ \tilde{d}_{j}(\tau, \theta), & \text { if } i=j-1 \text { ("death" of a mutant) } \\ 1-\left(\tilde{b}_{j}(\tau, \theta)+\tilde{d}_{j}(\tau, \theta)\right) & \text { if } j=i \text { ("no change") } \\ 0 & \text { otherwise. }\end{cases}
$$

Standard results on birth-death processes (e.g., Ewens, 2004, eq. 2.160, Mullon and Lehmann, 2014, eq. 8) show that when the initial state of the chain is one mutant, we have

$$
t_{i}(\tau, \theta)=\frac{1}{\tilde{d}_{1}(\tau, \theta)} \prod_{k=1}^{i-1} \frac{\tilde{b}_{k}(\tau, \theta)}{\tilde{d}_{k+1}(\tau, \theta)}
$$

In order to evaluate the $\tilde{b}_{k}$ 's and $\tilde{d}_{k}$ 's explicitly in terms of model parameter, we start to denote by $f_{k}\left(\theta^{\prime}, \theta\right)$ and $\mu_{k}\left(\theta^{\prime}, \theta\right)$, respectively, the fecundity and death-factor of a single type $\theta^{\prime} \in\{\tau, \theta\}$ individual when there are exactly $k$ mutants among its patch neighbors (see Box 2). Then, for the Moran process (Box 1) we have:

$\tilde{b}_{k}(\tau, \theta)=\frac{(N-k) \mu_{k}(\theta, \theta)}{k \mu_{k-1}(\tau, \theta)+(N-k) \mu_{k}(\theta, \theta)}\left[\frac{(1-m) k f_{k-1}(\tau, \theta)}{(1-m)\left[k f_{k-1}(\tau, \theta)+(N-k) f_{k}(\theta, \theta)\right]+m N f_{0}(\theta, \theta)}\right]$,

where the first factor is the probability that a resident is chosen to die and thus vacates a breeding spot and the second factor (term in square brackets) is the probability that this 
vacated breeding spot is occupied by a mutant. Hence, we have

$$
\begin{aligned}
\tilde{d}_{k}(\tau, \theta)=\left[1-\frac{(N-k) \mu_{k}(\theta, \theta)}{k \mu_{k}(\tau, \theta)+(N-k) \mu_{k}(\theta, \theta)}\right] & (1-m) k f_{k}(\tau, \theta) \\
& {\left[1-\frac{\left.(N-k) f_{k}(\theta, \theta)\right]+m N f_{0}(\theta, \theta)}{(1-m)\left[k f_{k}(\tau, \theta)+(N-\right.}\right] . }
\end{aligned}
$$

It now remains to express the $f_{k}$ 's and $d_{k}$ 's in terms of the fecundity and risk-factor functions $f\left(\theta_{i}, \boldsymbol{\theta}_{-i}, \phi\right)$ and $\mu\left(\theta_{i}, \boldsymbol{\theta}_{-i}, \phi\right)$. Owing to permutation invariance (and recalling the argument leading to eq. A-11), we have

$$
\begin{aligned}
& f_{k}\left(\theta^{\prime}, \theta\right)=\sum_{\boldsymbol{\theta}_{-i} \in S_{k}(\tau, \theta)}\left(\begin{array}{c}
N-1 \\
k-1
\end{array}\right)^{-1} f\left(\theta^{\prime}, \boldsymbol{\theta}_{-i}, 1_{\theta}\right), \text { for } k=1,2, \ldots, N-1 \\
& \mu_{k}\left(\theta^{\prime}, \theta\right)=\sum_{\boldsymbol{\theta}_{-i} \in S_{k}(\tau, \theta)}\left(\begin{array}{c}
N-1 \\
k-1
\end{array}\right)^{-1} \mu\left(\theta^{\prime}, \boldsymbol{\theta}_{-i}, 1_{\theta}\right), \text { for } k=1,2, \ldots, N-1 .
\end{aligned}
$$

On substitution of eqs. D-2-D-4 into lineage fitness (eq. A-14) along with the fitness function of the Moran process (Box 1 of the main text), we have all the elements to compute lineage fitness exactly under the Moran process for games of arbitrary complexity.

\section{Neutral distribution}

Setting $\tau=\theta$ in eqs. D-2-D-4, we can compute the full neutral distribution of types (Box 2), which gives

$$
p_{k}(\theta, \theta)=\frac{k m N}{m+N-1} \prod_{i=1}^{k-1} \frac{(1-m) i(N-i)}{(i+1)(N-(i+1)(1-m))},
$$

and on substitution into the expression for relatedness in Box 2 (by setting $\theta^{\prime}=\theta$ ) produces

$$
r(\theta, \theta)=\frac{1-m}{1-m+N m} .
$$

The same result can be obtained by using a standard (and simpler) identity-by-descent argument (e.g., Karlin, 1968; Rousset, 2004), implying that relatedness satisfies

$$
r(\theta, \theta)=(1-m)\left(\frac{1}{N}+\frac{(N-1)}{N} r(\theta, \theta)\right),
$$

whose solution results again in eq. D-7. 


\section{Cournot game example}

We here evaluate the second order conditions for the Cournot game discussed in section

"Uninvadability and maximizing behavior" of the main text under a Moran process when $N=2$ and with constant death rate. Substituting the fitness function of the Moran process (Box 1) and eqs. D-2-D-5 into lineage fitness (eq. A-14) along with the game payoff function (eq. 9 of the main text), allows us to readily compute (using Wolfram Mathematica 10) the second order condition (eq. B-22) at the unique element of $X_{\mathrm{D}}$ (see eq. 10 of the main text), which can be simplified to

$$
\left.\frac{\partial^{2} W(y, x)}{\partial y^{2}}\right|_{y=x=x^{*}}=-\frac{m[\beta(1-m)(2-m)+\gamma(3-m)] A}{(1+m)\left(A+\alpha^{2}(3-m)[\beta(1-m)+\gamma(3-m)]\right)},
$$

where $A=4[\beta(2-m)+\gamma(3-m)]^{2} \geq 0$.

Substituting the fitness function of the Moran process (Box 1), the game payoff function (eq. 9 of the main text), strategy $x^{*}$ (eq. 10 of the main text), and the associated relatedness (eq. D-7) into eq. B-23 yields

$$
\begin{aligned}
\left.\frac{\partial^{2} u_{\mathrm{A}}\left(x_{i}, \mathbf{x}^{(N-1)}, 1_{x}\right)}{\partial x_{i}^{2}}\right|_{x_{i}=x=x^{*}} & =-B\left[-2 \alpha^{2} \beta^{2}(1-m)^{3}\right. \\
& \left.+\gamma(3-m)\left(\gamma(3-m)^{2}\left(\alpha^{2}+4 \gamma\right)+4 \beta^{2}(2-m)^{2}+\beta(3-m) C\right)\right],
\end{aligned}
$$

where

$$
B=\frac{4 m[\gamma(3-m)+\beta(2-m)]^{2}}{(1+m)\left[(3-m)\left[\beta C+\gamma(3-m)\left(\alpha^{2}+4 \gamma\right)\right]+4 \beta^{2}(2-m)^{2}\right]^{2}} \geq 0
$$

and $C=8 \gamma(2-m)+\alpha^{2}(1-m) \geq 0$.

Finally, substituting eqs. D-2-D-5 into the expression for relatedness (Box 2) along with the game payoff function (eq. 9) allows us to compute (using Wolfram Mathematica 10) the relatedness effect of strategy $x^{*}$ (eq. 10 of the main text), which is

$$
\left.\frac{\partial r(y, x)}{\partial y}\right|_{y=x=x^{*}}=r_{1}\left(x^{*}, x^{*}\right)=0
$$




\section{Appendix E: constrained behavior}

Here we present a model of repeated interactions where there are mechanistic constraints between moment-to-moment actions. This allows us to illustrate how this changes the interpretation of maximizing behavior relative to the case where all actions can be optimal on the path of play. To that end, we consider a multi-move game where individuals have a memory of step one, and stay with the Moran process under $N=2$. A typical example is an infinitely repeated prisoner's dilemma where individuals can only react to the last action of their partner (e.g., McNamara et al., 1999; Taylor and Day, 2004; André and Day, 2007). The simplest setting therein is maybe provided by the so-called continuous prisoner's dilemma with linear reactive "strategies," where $a_{i}(t) \geq 0$ is the level of investment in cooperation by individual $i$ in social period $t$ of the repeated game, and is given by

$$
\left\{\begin{array}{l}
a_{1}(t)=\alpha+\theta_{1} a_{2}(t-1) \\
a_{2}(t)=\alpha+\theta_{2} a_{1}(t-1)
\end{array} \quad \text { for } t=1,2, \ldots\right.
$$

where $\alpha>0$ is an exogenous initial donation, and $a_{1}(0)=a_{2}(0)=\alpha$. Here $\theta_{i} \in \Theta=(0,1)$ represents the evolvable response slope of individual $i$ on the level of investment of its partner in the previous round. For this model, the decision rule is

$$
d_{i}\left(s_{i}(t)\right)=\alpha+\theta_{i} s_{i}(t) \text { with } s_{i}(t)=a_{-i}(t-1) \text { and } s_{i}(0)=0 \text {. }
$$

Given some material payoffs in each round $t=0,1,2, \ldots$ uninvadability of $\theta$ can be evaluated, for example, in terms of the long-run average material payoff. This average is well-defined, since both individuals' actions increase monotonically over social time and converge to the within-period action pair

$$
\left\{\begin{array}{l}
a_{1}^{*}=\alpha\left(1+\theta_{1}\right) /\left(1-\theta_{1} \theta_{2}\right) \\
a_{2}^{*}=\alpha\left(1+\theta_{2}\right) /\left(1-\theta_{1} \theta_{2}\right) .
\end{array}\right.
$$

Hence, if the material payoffs in each time period of the repeated interaction are given by $\psi\left(a, a^{\prime}\right)$, where $a$ is own action and $a^{\prime}$ the other individual's action, then the long-run average material payoff within the demographic time period to an individual with trait $\theta$ interacting with an individual with trait $\theta^{\prime}$ is

$$
\pi\left(\theta, \theta^{\prime}, \phi\right)=\psi\left[\frac{\alpha(1+\theta)}{1-\theta^{\prime} \theta}, \frac{\alpha\left(1+\theta^{\prime}\right)}{1-\theta^{\prime} \theta}\right] .
$$


For the sake of illustration, suppose the function $\psi$ is linear-quadratic:

$$
\psi\left(a, a^{\prime}\right)=\beta a a^{\prime}-\gamma a^{2}
$$

for some $\beta, \gamma>0$. Substituting the resulting payoff function into lineage payoff (eq. C-9) and computing the first-order condition for a type $\theta \in(0,1)$ to be a locally uninvadable shows that $X_{\mathrm{UW}}$ is a singleton set with unique element

$$
\theta^{*}=\frac{1-(2-m)(\beta / \gamma-1)}{1+(2-m)(\beta / \gamma-1)} .
$$

The necessary second-order condition for uninvadability is $\gamma \leq \beta$ for a panmictic population $(m=1)$, and $m(2 \beta-\gamma)^{5} /(\beta-\gamma)^{3} \leq 0$ for $m$ close to zero. The second-order condition is complicated for intermediate values of $m$. But since a necessary condition for $\theta^{*} \in(0,1)$ is that $\gamma<\beta$, the two boundary cases are sufficient to illustrate the fact that limited dispersal tends to destabilize the candidate uninvadable point; $\theta^{*}$ is uninvadable in a panmictic population, while for strong population structures $\theta^{*}$ is invadable.

By Result 6 , the locally uninvadable type $\theta^{*}$ is also the unique symmetric Nash equilibrium strategy $x^{*}$ when individuals' goal function is $u_{\mathrm{C}}$ and $X=\Theta=(0,1)$. But is it behaviorally/biologically reasonable to interpret the reaction slope as a strategy $x^{*}$, chosen by individuals? Under the infinitely repeated prisoners' dilemma (eqs. E-1-E-4), the reaction slope $\theta$ determines the decision rule of individuals (eq. E-2). From a game-theoretic viewpoint, this constrained repeated prisoners' dilemma is a one-shot game, where each player only has one choice, namely what reaction slope $x \in X=\Theta=(0,1)$ to use throughout the whole social interaction. Hence, the reaction slope is now the player's strategy in a simultaneous-move one-shot game with material payoff from playing strategy $x^{\prime}$ against strategy $x$ given by eq. E- 4 with $\theta^{\prime}$ replaced by $x^{\prime}$ and $\theta$ by $x$. The interpretation in terms of maximizing behavior is then that individuals choose how strongly to react to the other player's last action, within the given class of affine functions.

\section{References}

Ajar, E. 2003. Analysis of disruptive selection in subdivided populations. BMC Evolutionary Biology 3:22. 
André, J. B. and T. Day. 2007. Perfect reciprocity is the only evolutionarily stable strategy in the continuous iterated prisoner's dilemma. Journal of Theoretical Biology 247:11-22.

Crow, J. F. and M. Kimura. 1970. An Introduction to Population Genetics Theory. Harper and Row, New York.

Ewens, W. J. 2004. Mathematical Population Genetics. Springer-Verlag, New York.

Frank, S. A. 1998. Foundations of Social Evolution. Princeton University Press, Princeton, NJ.

Gillespie, J. H. 2004. Population Genetics: a Concise Guide. Johns Hopkins, Baltimore \& London.

Grinstead, C. M. and J. L. Snell. 1997. Introduction to Probability. American Mathematical Society, Providence, RI, second edn.

Karlin, S. 1968. Equilibrium behavior of population genetic models with non-random mating: part II: pedigrees, homozygosity and stochastic models. Journal of Applied Probability $5: 487-566$.

Karlin, S. and H. M. Taylor. 1975. A First Course in Stochastic Processes. Academic Press, San Diego.

Lehmann, L. and F. Rousset. 2009. Perturbation expansions of multilocus fixation probabilities for frequency-dependent selection with applications to the Hill-Robertson effect and to the joint evolution of helping and punishment. Theoretical Population Biology 76:35-51.

Lessard, S. 2009. Diffusion approximations for one-locus multi-allele kin selection, mutation and random drift in group-structured populations: a unifying approach to selection models in population genetics. Journal of Mathematical Biology 59:659-696.

Massol, F., V. Calcagno, and J. Massol. 2009. The metapopulation fitness criterion: proof and perspectives. Theor Popul Biol 75:183-200.

McNamara, J. M., C. E. Gasson, and A. I. Houston. 1999. Incorporating rules for responding into evolutionary games. Nature 401:368-71. 
Metz, J. and M. Gyllenberg. 2001. How should we define fitness in structured metapopulation models? Including an application to the calculation of evolutionary stable dispersal strategies. Proceedings of the Royal Society of London Series B-Biological Sciences 268:499-508.

Mullon, C., L. Keller, and L. Lehmann. In prep. Evolutionary stability of multiple traits in subdivided populations .

Mullon, C. and L. Lehmann. 2014. The robustness of the weak selection approximation for the evolution of altruism against strong selection. Journal of Evolutionary Biology $27: 2272-2282$.

Rousset, F. 2004. Genetic Structure and Selection in Subdivided Populations. Princeton University Press, Princeton, NJ.

Roze, D. and F. Rousset. 2003. Selection and drift in subdivided populations: A straightforward method for deriving diffusion approximations and applications involving dominance, selfing and local extinctions. Genetics 165:2153-2166.

Taylor, P. D. and T. Day. 2004. Stability in negotiation games and the emergence of cooperation. Proceedings of the Royal Society of London Series B-Biological Sciences 271:669-674.

Thieme, H. R. 2009. Spectral bound and reproduction number for infinite-dimensional population structure and time heterogeneity. SIAM Journal of applied mathematics 70:188-211.

Wakano, J. Y. and L. Lehmann. 2014. Evolutionary branching in deme-structured populations. Journal of Theoretical Biology 351:83-95.

Wild, G. 2011. Inclusive fitness from multitype branching processes. Bulletin of Mathematical Biology 73:1028-51. 